Article

\title{
Synthesis of New Furothiazolo Pyrimido Quinazolinones from Visnagenone or Khellinone and Antimicrobial Activity
}

\author{
Ameen Ali Abu-Hashem 1,2 (D) \\ 1 Photochemistry Department (Heterocyclic Unit), National Research Centre, Dokki, Giza1 2622, Egypt; \\ aminaliabuhashem@yahoo.com; Tel.: +2-01225211700 or +966-591363915; Fax: + 202-33370931 \\ 2 Chemistry Department, Faculty of Science, Jazan University, 2097 Jazan, Saudi Arabia
}

Academic Editor: Josef Jampilek

Received: 5 October 2018; Accepted: 24 October 2018; Published: 27 October 2018

\begin{abstract}
Substituted-6-methyl-1-thioxo-1,2-dihydro-3H-furo[3,2-g]pyrimido[1,6-a]quinazolin-3ones $(\mathbf{5} \mathbf{a}, \mathbf{b})$ were synthesized from condensation of visnagenone $(\mathbf{2} \mathbf{a})$ or khellinone $(\mathbf{2} \mathbf{b})$ with 6 amino-thiouracil (3) in dimethylformamide or refluxing of (4a) or (4b) in dimethylformamide. Hence, compounds $(\mathbf{5} \mathbf{a}, \mathbf{b})$ were used as the starting materials for preparing many new heterocyclic compounds such as; furo[3,2-g]pyrimido[1,6-a]quinazoline $(\mathbf{6 a}, \mathbf{b})$, furo[3,2-g]thiazolo[ $\left[2^{\prime}, 3^{\prime}: 2,3\right]$ pyrimido[1,6-a]quinazolinone $(7 \mathbf{a}, \mathbf{b})$, substituted-benzylidene-furo[3,2-g]thiazolo $\left[2^{\prime}, 3^{\prime}: 2,3\right]$ pyrimido [1,6-a]quinazoline-3,5-dione (8a-f), 3-oxo-furo[3,2-g]pyrimido[1,6-a]quinazoline-pentane-2,4-dione $(\mathbf{9 a}, \mathbf{b}), 1$-(pyrazole)-furo[3,2-g]pyrimido[1,6-a]quinazolinone (10a,b), 2-(oxo or thioxo)-pyrimidinefuro[3,2-g]pyrimido[1,6-a]quinazolinone (11a-d), 1-(methylthio)-furo[3,2-g]pyrimido[1,6-a] quinazolinone (12a,b), 1-(methyl-sulfonyl)-furo[3,2-g]pyrimido[1,6-a]quinazolinone (13a,b) and 6-methyl-1-((piperazine) or morpholino)-3H-furo[3,2-g]pyrimido[1,6-a]quinazolin-3-one (14a-d). The structures of the prepared compounds were elucidated on the basis of spectral data (IR, ${ }^{1} \mathrm{H}-\mathrm{NMR}$, ${ }^{13} \mathrm{C}-\mathrm{NMR}, \mathrm{MS}$ ) and elemental analysis. Antimicrobial activity was evaluated for the synthesized compounds against Gram-positive, Gram-negative bacteria and fungi. The new compounds, furothiazolo pyrimido quinazolines $\mathbf{8 a - f}$ and 11a-d displayed results excellent for growth inhibition of bacteria and fungi.
\end{abstract}

Keywords: visnagenone; khellinone; quinazolinone; pyrimidine; thiazole; pyrazole; furane; antimicrobial activity

\section{Introduction}

Physicians confirm the usefulness of adding many naturally occurring drugs. Furochromones (visnagin and khellin) compounds are natural products extracted from the plant of Ammi visnaga Lam. and visnagin and khellin are used as therapy for kidney, bladder stones, diuretic infusions $[1,2]$ and are considered essential components of many drugs [3]. As well, furochromone derivatives are reported as anti-atherosclerotic, antineoplastic, anti-gastric, anti-anaphylactic and are used in the treatment of urolithiasis, hypertriglyceridemia [4,5] and vitiligo [6]. Also, the visnaginone derivatives were synthesized using different procedures and elucidated efficient antimicrobial activities [7,8]. Furthermore, furochromones have been used to treat pain in the renal colic [9] and possesses coronary vasodilating activity $[10,11]$. Moreover, khellin and visnagin have been used in the photo-chemotherapeutic treatment of vitiligo and psoriasis [12], photoreaction with DNA [13] and Khellin displayed important epidermal growth factor receptor (EGFR) inhibitory activity [14]. Benzofurans and furochromones [15-18] are very exciting heterocycles, which are omnipresent in nature and display a wide range of pharmacological activities. Recently, furochromones 
and benzofurans derivatives are used as in antiviral and anticancer activities [19,20]. When fused of furochromones with pyrimidine, quinoxaline and pyrazole derivatives showed anti-inflammatory and analgesic activities [21], the cytotoxic activity [22] and used in the protection of DNA [23]. Additionally, some of the moieties heterocyclic such as, chalcones [24], thiazolidinones [25], Mannich bases [26], sulfonamides [27], and isoxazole [28], too, showed several of biological activity with benzofuran derivatives (visnagenone, khellinone) (Figure 1).<smiles>[R]c1c2occc2c(OC)c2c(=O)cc(C)oc12</smiles>

visnagin (1a), $\mathrm{R}=\mathrm{H}$ khellin (1b), $\mathrm{R}=\mathrm{OCH}_{3}$<smiles>[R]c1c(O)c(C(C)=O)c(OC)c2ccoc12</smiles>

2

visnagenone (2a), $\mathrm{R}=\mathrm{H}$ khellinone (2b), $\mathrm{R}=\mathrm{OCH}_{3}$<smiles>[R]c1c2occc2c(OC)c2c(=O)cc(CO[C@H]3O[C@H](CO)[C@@H](O)[C@H](O)[C@H]3O)oc12</smiles>

Khellol glucoside, $\mathrm{R}=\mathrm{OCH}_{3}$

Figure 1. Chemical structure of furochromone derivatives.

An extension of our work on the preparation of novel heterocyclic compounds resultant from the naturally occurring visnagin and khellin [21-23,29], we synthesized and described several derivatives which contained a benzofuran moiety (visnaginone, khellinone) incorporated with thiazole, pyrimidine, pyrazole, and quinazolinone derivatives. The antimicrobial activity of the prepared compounds were evaluated.

\section{Results and Discussion}

\subsection{Synthesis}

In the present work, the natural furochromones (visnagin 1a or khellin $\mathbf{1 b}$ ) are highly sensitive to alkali. Hence, aqueous alkaline hydrolysis of $\mathbf{1 a}$ and $\mathbf{1} \mathbf{b}$ using potassium hydroxide with heating and stirring at $40-50{ }^{\circ} \mathrm{C}$ for $1-2 \mathrm{~h}$ lead to form visnagenone $\mathbf{2 a}$ or khellinone $\mathbf{2} \mathbf{b}$, respectively [22,23]. Moreover, heating under reflux cyclic alfa, beta-unsaturated ketones (visnagenone 2a or khellinone 2b) with 6-aminothiouracil (3) in dimethylformamide solution [21,22] with stirring for 4-6 h or 14-16 h under (TLC) afforded new compounds respectively, 6-((1-(6-hydroxy-(4-methoxy or 4,7-dimethoxy)-benzofuran-5-yl)ethylidene)amino)-2-thioxo-2,3-dihydropyrimidin-4(1H)-one (4a,b) and (7-methoxy or 7,11-dimethoxy)-6-methyl-1-thioxo-1,2-dihydro-3H-furo[3,2-g]pyrimido[1,6-a] quinazolin-3-one $(\mathbf{5 a}, \mathbf{b})$ in good yield obtained. Also, another method, stirring under reflux of $4 \mathbf{a}$ or $4 \mathbf{b}$ in dimethylformamide solution for 8-10 $\mathrm{h}$ afforded the same products $\mathbf{5 a}$ and $\mathbf{5 b}$, respectively. The ${ }^{1} \mathrm{H}-\mathrm{NMR}$ spectrum of compound 4a showed three singlet broad signal at 10.80,11.19 and $16.11 \mathrm{ppm}$ corresponding to the three protons of the $(2 \mathrm{NH})$ and one $(\mathrm{OH})$ groups, which were $\mathrm{D}_{2} \mathrm{O}$ exchangeable and ${ }^{1} \mathrm{H}-\mathrm{NMR}$ spectrum of compound 5 a showed one singlet broad signal at 11.20 ppm conforming to the one proton of the one $(\mathrm{NH})$ group $\left(\mathrm{D}_{2} \mathrm{O}\right.$ exchangeable) and the mass spectra of $4 \mathbf{a}, 4 \mathbf{b}, \mathbf{5} \mathbf{a}$ and 5b showed molecular ion peaks at $m / z 331\left(\mathrm{M}^{+}, 100 \%\right)$ and $361\left(\mathrm{M}^{+}, 100 \%\right), 313\left(\mathrm{M}^{+}, 100 \%\right), 343\left(\mathrm{M}^{+}\right.$, $100 \%$ ) respectively (Scheme 1). 
<smiles>[R]c1c(O)c(C(C)=O)c(OC)c2ccoc12</smiles>

2a, $\mathrm{R}=\mathrm{H} ; \mathbf{2} \mathbf{b}, \mathrm{R}=\mathrm{OCH}_{3}$

aq. $\mathrm{KOH} / \mathrm{HCl}$<smiles>[R]c1c2occc2c(OC)c2c(=O)cc(C)oc12</smiles>

$1 \mathbf{a}, \mathbf{b}$

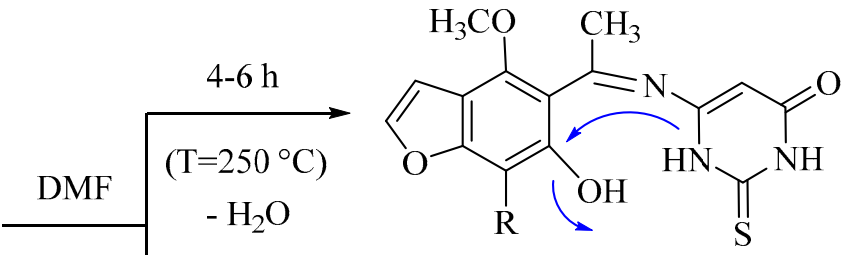

$\mathbf{4 a}, \mathrm{R}=\mathrm{H} ; \mathbf{4 b}, \mathrm{R}=\mathrm{OCH}_{3}$

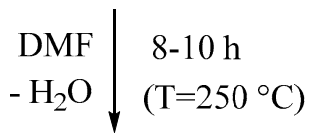

14-16 h

$\left(\mathrm{T}=260^{\circ} \mathrm{C}\right)$

$-2 \mathrm{H}_{2} \mathrm{O}$

visnagen (1a), $\mathrm{R}=\mathrm{H}$

khellin (1b), $\mathrm{R}=\mathrm{OCH}_{3}$<smiles>[R]c1c2occc2c(OC)c2c(C)nc3cc(=O)[nH]c(=S)n3c12</smiles>

5a, $\mathrm{R}=\mathrm{H} ; \mathbf{5} \mathbf{b}, \mathrm{R}=\mathrm{OCH}_{3}$

Scheme 1. Synthesis of furopyrimido quinazolinones from visnagenone and khellinone derivatives.

In this research, simple and convenient methods to the syntheses of furopyrimido quinazoline, furothiazolo pyrimido quinazolinone and furothiazolo pyrimido quinazolinone benzylidene derivatives [30]. Thus, refluxing of $5 \mathbf{a}$ or $\mathbf{5} \mathbf{b}$ with chloroacetic acid in glacial acetic acid/acetic anhydride and anhydrous sodium acetate for $3-5 \mathrm{~h}$ or $13-15 \mathrm{~h}$ with control via (TLC) to give new compounds followed by, 2-((7-methoxy or 7,11-dimethoxy)-6-methyl-3-oxo-3H-furo[3,2-g]pyrimido[1,6-a] quinazolin-1-yl)thio)acetic acid (6a,b) and (9-methoxy or 9,13-dimethoxy)-8-methyl-2-hydro-5H,14aHfuro[3,2-g]thiazolo[2', $\left.3^{\prime}: 2,3\right]$ pyrimido[1,6-a]quinazoline-3,5-dione $(\mathbf{7 a}, \mathbf{b})$ in high yields. Moreover, the boiling of $\mathbf{6 a}$ or $\mathbf{6 b}$ in dimethylformamide solution resulted in the same formation of $\mathbf{7 a}$ and $7 \mathbf{b}$. The IR spectrum of compounds $\mathbf{6 a}$ and $\mathbf{6 b}$ exposed the presence of broad band absorption at $3340-3350 \mathrm{~cm}^{-1}$ indicative of one $(\mathrm{OH})$ group and $\mathbf{6 a}=1745,1684 \mathrm{~cm}^{-1}$ and $\mathbf{6 b}=1748$, $1681 \mathrm{~cm}^{-1}$ of the two carbonyl groups. The ${ }^{1} \mathrm{H}-\mathrm{NMR}$ spectrum of $6 \mathbf{a}$ showed a singlet broad signal at $13.70 \mathrm{ppm}$ corresponding to the one protons of the one $(\mathrm{OH})$ group, which were $\mathrm{D}_{2} \mathrm{O}$ exchangeable. Also, ${ }^{1} \mathrm{H}-\mathrm{NMR}$ spectrum of $\mathbf{7 b}$ displayed a five singlet signal at $\delta 2.33,3.97,4.24$, 5.62 and $7.39 \mathrm{ppm}$ conforming to the thirteen protons of the methyl, two methoxy, $\left(\mathrm{CH}_{2}\right),(\mathrm{CH}$, thiazole) and ( $\mathrm{CH}$, pyrimidine) groups, respectively. As well as using one-pot synthesis as follows: when a ternary mixture of $\mathbf{5} \mathbf{a}$ or $\mathbf{5} \mathbf{b}$, chloroacetic acid and a proper aldehyde namely; benzaldehyde, 4-chlorobenzaldehyde or 4-methoxybenzaldehyde respectively, was heated under reflux in a mixture of acetic acid and acetic anhydride in the presence of anhydrous sodium acetate afforded the conforming 2-(substituted-benzylidene)-9,(substituted)-methoxy-8-methyl-2-hydro-5H,14aH-furo[3,2-g]thiazolo $\left[2^{\prime}, 3^{\prime}: 2,3\right]$ pyrimido[1,6-a]quinazoline-3,5-dione (8a-f) in high yields. In another route, we obtained on the same compounds $(\mathbf{8} \mathbf{a}-\mathbf{f})$ via refluxing of $\mathbf{7 a}$ or $\mathbf{7 b}$ with appropriate aromatic aldehyde in dioxane solution containing a catalyst amount of piperidine for 10-12 h. ${ }^{1} \mathrm{H}-\mathrm{NMR}$ spectrum of 8a showed six singlets at 2.30, 3.91, 5.59, 7.32, 7.85 and 8.05 ppm conforming to the 10 protons of the methyl, methoxy, thiazole proton, pyrimidine proton, phenyl proton and $(\mathrm{CH})$ proton groups, respectively and two doublet signals at $6.75,7.72 \mathrm{ppm}$ of the two protons $(J=2.30 \mathrm{~Hz}$, furan). The structures assignments for compounds were established on their elemental analysis and spectral (IR, ${ }^{1} \mathrm{H},{ }^{13} \mathrm{C}-\mathrm{NMR}$, and MS) data are shown in the experimental section (Scheme 2). 
<smiles>[R]c1c2occc2c(OC)c2c(C)nc3cc(=O)[nH]c(=S)n3c12</smiles>

5a, $\mathrm{R}=\mathrm{H} ; \mathbf{5 b}, \mathrm{R}=\mathrm{OCH}_{3}$

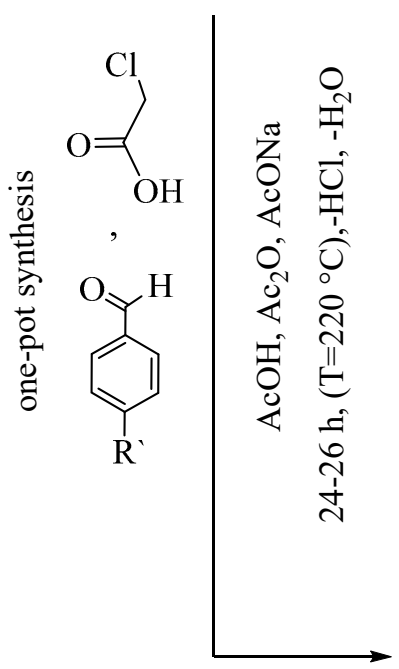

$8 \mathrm{a}, \mathrm{R}=\mathrm{H} ; \quad \mathrm{R}^{\prime}=\mathrm{H}$

$8 \mathrm{~b}, \mathrm{R}=\mathrm{H} ; \quad \mathrm{R}=\mathrm{Cl}$

$8 \mathrm{c}, \mathrm{R}=\mathrm{H} ; \quad \mathrm{R}=\mathrm{OCH}_{3}$

$8 \mathrm{~d}, \mathrm{R}=\mathrm{OCH}_{3} ; \mathrm{R}=\mathrm{H}$

$8 \mathrm{e}, \mathrm{R}=\mathrm{OCH}_{3} ; \mathrm{R}=\mathrm{Cl}$

8f, $\mathrm{R}=\mathrm{OCH}_{3} ; \mathrm{R}=\mathrm{OCH}_{3}$<smiles>[R]c1c2occc2c(OC)c2c(C)nc3cc(=O)nc(SCC(=O)O)n3c12</smiles>

6a, $\mathrm{R}=\mathrm{H} ; \mathbf{6} \mathbf{b}, \mathrm{R}=\mathrm{OCH}_{3}$

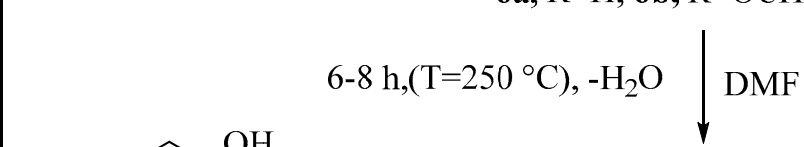

$$
\mathrm{Cl} \bigwedge_{\mathrm{O}}^{\mathrm{OH}}
$$

$\mathrm{AcOH}, \mathrm{Ac}_{2} \mathrm{O}, \mathrm{AcONa}$

13-15 h, $\left(\mathrm{T}=220^{\circ} \mathrm{C}\right),-\mathrm{HCl}$

$$
-\mathrm{H}_{2} \mathrm{O}
$$<smiles>[R]c1c2c(c(OC)c3ccoc13)C(C)=NC1=CC(=O)N3C(=O)CS[C@H]3N12</smiles>

7a, $\mathrm{R}=\mathrm{H} ; \mathbf{7 b}, \mathrm{R}=\mathrm{OCH}_{3}$<smiles></smiles><smiles>[R]c1ccc(C=O)cc1</smiles>

dioxane / piperidine

$10-12 \mathrm{~h},\left(\mathrm{~T}=230^{\circ} \mathrm{C}\right)$

Scheme 2. Synthesis of furothiazolopyrimidoquinazolinone derivatives.

Alkylation of an ethanolic potassium hydroxide solution of $\mathbf{5 a}$ or $\mathbf{5 b}$ with 3-chloro-pentane2,4-dione (3-chloroacetylacetone) [30], yielded the 3-(((7-methoxy or 7,11-dimethoxy)-6-methyl-3-oxo-3Hfuro[3,2-g]pyrimido[1,6-a]quinazolin-1-yl)thio)pentane-2,4-dione $(\mathbf{9 a}, \mathbf{b})$ in high yield. The IR spectra of $\mathbf{9 a}$ and $\mathbf{9 b}$ showed a strong absorption bands at $\mathbf{9 a}=1725,1721,1682 \mathrm{~cm}^{-1}$ and $\mathbf{9 b}=1728,1722$, $1684 \mathrm{~cm}^{-1}$ characteristic to three carbonyl groups, respectively. The ${ }^{13} \mathrm{C}-\mathrm{NMR}$ (DMSO- $d_{6}, \mathrm{ppm}$ ) of 9a showed signals at 68.2, 91.3 and 96.5 for three carbon atoms of $(\mathrm{CH}),(\mathrm{CH}$, phenyl) and $(\mathrm{CH}$, pyrimidine) and signals at 168.6, 184.5 and 188.2 three carbon atoms of the three carbonyl groups, and the molecular ion peaks of $\mathbf{9 a}$ and $\mathbf{9 b}$ at $\mathrm{m} / \mathrm{z} 411$ (95\%) and 441 (90\%), respectively. On the other hand, compounds $\mathbf{9 a}$ and $\mathbf{9 b}$, as a typical 1,3-diketone condensation with each of hydrazine hydrate, urea and thiourea to afford the corresponding 1-((3,5-dimethyl-1H-pyrazol-4-yl)thio)-(7-methoxy or 7,11-dimethoxy)-6-methyl-3H-furo[3,2-g]pyrimido[1,6-a]quinazolin-3-one (10a,b) and 1-((4,6-dimethyl2-(oxo or thioxo)-1,2-dihydropyrimidin-5-yl)thio)-(7-methoxy or 7,11-dimethoxy)-6-methyl-3H-furo[3,2-g] pyrimido[1,6-a]quinazolin-3-one (11a-d), respectively. IR spectrum of 10a and 10b showed absorption of a broad band at $3380-3360 \mathrm{~cm}^{-1}$ corresponding to NH group. Also, the compounds 11a-d exhibited absorption bands at $3390-3370 \mathrm{~cm}^{-1}$ for $\mathrm{NH}$ group were detected in the IR spectrum. ${ }^{1} \mathrm{H}-\mathrm{NMR}$ spectrum of 10a showed one singlet at $11.50 \mathrm{ppm}$ corresponding to the one proton of $\mathrm{NH}$ group and compound 11a revealed one singlet at $10.60 \mathrm{ppm}$ for one proton of $\mathrm{NH}$ group $\left(\mathrm{D}_{2} \mathrm{O}\right.$ exchangeable). All new compounds 
were proven by elemental and spectral analysis (IR, ${ }^{1} \mathrm{H},{ }^{13} \mathrm{C}-\mathrm{NMR}$ and MS) which is mentioned in the experimental part (Scheme 3).<smiles>[R]Oc1n[nH]c(C)c1Sc1nc(=O)cc2nc(C)c3c(OC)c4ccoc4c([R])c3n12</smiles>

Scheme 3. Synthesis of pyrazole, pyrimidine, -furopyrimido quinazolinone derivatives.

Alkylation of an ethanolic potassium hydroxide solution of $\mathbf{5 a}$ or $\mathbf{5 b}$ with methyl-iodide yielded the (7-methoxy or 7,11-dimethoxy)-6-methyl-1-(methylthio)-3H-furo[3,2-g]pyrimido[1,6-a] quinazolin-3-one (12a,b). Assignment of structures $\mathbf{1 2 a}$ and $\mathbf{1 2 b}$ to the reaction products is based on correct elemental analysis and IR, NMR spectroscopy are in agreement with the structure. Thus, ${ }^{1} \mathrm{H}-\mathrm{NMR}$ spectrum of $\mathbf{1 2 a}$ or $\mathbf{1 2} \mathbf{b}$ showed one singlet signal at 2.80 or $2.88 \mathrm{ppm}$ indicative of three protons to $\left(\mathrm{SCH}_{3}\right)$ group and the molecular ion peaks of $\mathbf{1 2 a}$ or $\mathbf{1 2} \mathbf{b}$ displayed at $m / z 327\left(\mathrm{M}^{+}, 100 \%\right)$ and $357\left(\mathrm{M}^{+}, 100 \%\right)$, respectively. Moreover, oxidation of $\mathbf{1 2} \mathbf{a}$ or $\mathbf{1 2} \mathbf{b}$ with hydrogen peroxide in acetic acid yielded the (7-methoxy or 7,11-dimethoxy)-6-methyl-1-(methylsulfonyl)-3H-furo[3,2-g] pyrimido[1,6-a]quinazolin-3-one $(\mathbf{1 3} \mathbf{a}, \mathbf{b})$. The IR spectrum of 13a exposed the presence of two bands at $1162,1340 \mathrm{~cm}^{-1}$ corresponding to $\left(\mathrm{SO}_{2}\right)$ group, and ${ }^{1} \mathrm{H}-\mathrm{NMR}$ spectrum of 13a exhibited one singlet at 2.95 agreeing to the three protons of $\left(\mathrm{SO}_{2} \mathrm{CH}_{3}\right)$ group. Furthermore, heating under refluxing compounds $\mathbf{1 2} \mathbf{a}$ or $\mathbf{1 2} \mathbf{b}$ with secondary aliphatic amines [21,22,30], namely piperazine or morpholine in methanol, produced the (7-methoxy or 7,11-dimethoxy)-6-methyl-1-((piperazin-1-yl) or morpholino)-3H-furo[3,2-g]pyrimido[1,6-a]quinazolin-3-one (14a-d). IR spectrum of 14a displayed absorption bands at $3390,1687 \mathrm{~cm}^{-1}$ conforming to $\mathrm{NH}$ and carbonyl group, respectively. Additionally, the ${ }^{1} \mathrm{H}-\mathrm{NMR}$ spectrum of $14 \mathrm{c}$ displayed a singlet broad signal at $10.35 \mathrm{ppm}$ conforming to the one proton of the one $(\mathrm{NH})$ group, which was $\mathrm{D}_{2} \mathrm{O}$ exchangeable. The mass spectrum of $\mathbf{1 3} \mathbf{a}, \mathbf{1 3} \mathbf{b}, \mathbf{1 4} \mathbf{a}, \mathbf{1 4} \mathbf{b}$, 14c and 14d revealed molecular ion peaks at $m / z 359\left(\mathrm{M}^{+}, 84 \%\right), 389\left(\mathrm{M}^{+}, 80 \%\right), 365\left(\mathrm{M}^{+}, 83 \%\right), 366\left(\mathrm{M}^{+}\right.$, $78 \%), 395\left(\mathrm{M}^{+}, 76 \%\right)$ and $396\left(\mathrm{M}^{+}, 74 \%\right)$, respectively (Scheme 4$)$. 


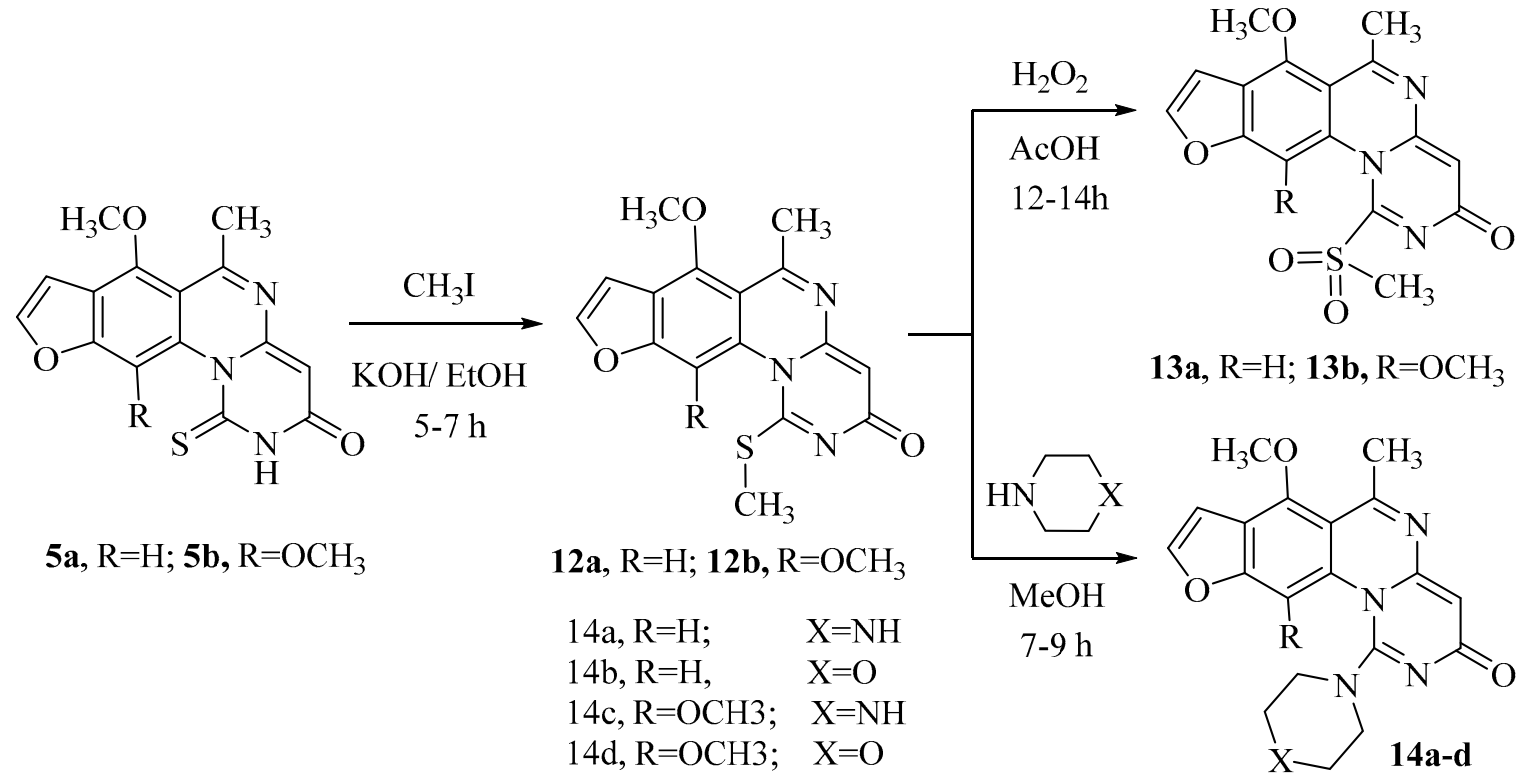

Scheme 4. Synthesis of piperazine, morpholine, -furopyrimido-quinazolinone derivatives.

\subsection{Biological Screening}

The antimicrobial activity of the compounds was tested in vitro and the results are shown in Tables 1 and 2. Some of these compounds indicated highest antimicrobial activity, comparable to cefotaxime sodium (MIC $=2-5 \mu \mathrm{mol} \mathrm{mL}^{-1}$ ). Compounds $\mathbf{8 a}-\mathbf{f}, \mathbf{1 1} \mathbf{a}-\mathbf{d}$ and 10a-b displayed potent antimicrobial activity against Gram-positive bacteria; Staphylococcus aureus (ATCC ${ }^{\circledR} 6538^{\mathrm{TM}}$ ), Streptococcus pyogenes (ATCC ${ }^{\circledR} 19615^{\mathrm{TM}}$ ) and Gram-negative bacteria; Escherichia coli (ATCC ${ }^{\circledR} 25922^{\mathrm{TM}}$ ), Klebsiella pneumoniae (ATCC ${ }^{\circledR} 10031^{\mathrm{TM}}$ ). Also, another compounds; 14a-d, $\mathbf{7 a} \mathbf{a}-\mathbf{b}$ and $\mathbf{9 a}-\mathbf{b}$ exhibited moderate antimicrobial activities. The MIC values in $\mu \mathrm{mol} \mathrm{mL} \mathrm{m}^{-1}$ of these compounds were as the following, 8a-f $(1-8), \mathbf{1 1} \mathbf{a}-\mathbf{d}(6-10), \mathbf{1 0} \mathbf{a}-\mathbf{b}(9-12)$. Compounds 8a-f and 11a-d exposed too higher antifungal activity with MIC in $\mu \mathrm{mol} / \mathrm{cm}^{3}$ of $\mathbf{8 a}-\mathbf{f}(2-9)$ and 11a-d (7-12) whose results were comparable with the positive control, nystatin (MIC 2-4 $\mu \mathrm{mol} \mathrm{mL}{ }^{-1}$ ). Some of compounds revealed moderate anti-fungal activity when compared with the nystatin (MIC $2-4 \mu \mathrm{mol} \mathrm{mL}^{-1}$ ): 10a-b (10-14), 14a-d (12-18), 7a-b (15-20) and 9a-b (17-22). The tested fungi were Aspergillus niger (ATCC ${ }^{\circledR} 16888^{\mathrm{TM}}$ ), Alternaria alternate, Curvularia lunata and Candida albicans (ATCC ${ }^{\circledR} 10231^{\mathrm{TM}}$ ). Thru comparing the observed antimicrobial activities of the furopyrimido quinazolinones, furothiazolo pyrimido quinazolinones obtained in this study to their structures, the (SAR's) were suggested; the presence of the functional groups linked with visnagenone $\mathbf{2 a}$ or khellinone $\mathbf{2} \mathbf{b}$ derivatives, such as thioxo, methyl, hydroxyl, methoxy, amino, chloro, substituted-benzylidene, thiazole, quinazoline, pyrimidine, pyrazole, methylsulfonyl, piperazine and morpholine moieties. Remainder the compounds demonstrated a weak activity compared to the antimicrobial activity of the standard drugs (cefotaxime sodium and nystatin). The thiazolopyrimidine derivatives possessing verified to be effective as antimicrobial agents in previous articles [7,8]. Also, they were active against Staphylococcus aureus, E. coli, and Klebsiella pneumoniae $[7,31]$ and they were also effective against Streptococcus pyogenes and species of fungi, namely, Aspergillus niger, Candida albicans [7,32]. In this work and previous reports, most bacteria and fungi types effected susceptible to this class of heterocyclic compounds [33-35]. Moreover, previous results and our findings corroboration the promising antimicrobial activity of furothiazolopyrimidoquinazolinone derivatives which can be developed to higher antimicrobial activities 
Table 1. A minimum inhibitory concentration of the compounds against bacteria.

\begin{tabular}{|c|c|c|c|c|}
\hline \multicolumn{5}{|c|}{$\operatorname{MIC}\left(\mu \mathrm{mol} \mathrm{mL^{-1 } )}\right.$} \\
\hline \multirow{3}{*}{ Compounds } & \multicolumn{4}{|c|}{ Microorganisms } \\
\hline & \multicolumn{2}{|c|}{ Gram-Positive Bacteria } & \multicolumn{2}{|c|}{ Gram-Negative Bacteria } \\
\hline & $\begin{array}{c}\text { Staphylococcus } \\
\text { Aureus }\end{array}$ & $\begin{array}{l}\text { Streptococcus } \\
\text { Pyogenes }\end{array}$ & E. coli & $\begin{array}{c}\text { Klebsiella } \\
\text { Pneumoniae }\end{array}$ \\
\hline $1 \mathbf{a}$ & 34 & 33 & 31 & 30 \\
\hline $1 b$ & 33 & 32 & 30 & 29 \\
\hline $2 a$ & 31 & 30 & 29 & 28 \\
\hline $2 b$ & 30 & 29 & 28 & 27 \\
\hline 3 & 37 & 35 & 34 & 33 \\
\hline $4 a$ & 29 & 28 & 27 & 26 \\
\hline $4 b$ & 28 & 27 & 26 & 25 \\
\hline $5 a$ & 27 & 26 & 25 & 24 \\
\hline $5 b$ & 26 & 25 & 24 & 23 \\
\hline $6 \mathbf{a}$ & 23 & 22 & 21 & 20 \\
\hline $6 b$ & 22 & 21 & 20 & 19 \\
\hline $7 \mathbf{a}$ & 17 & 16 & 15 & 14 \\
\hline $7 \mathbf{b}$ & 16 & 15 & 14 & 13 \\
\hline $8 a$ & 8 & 7 & 7 & 5 \\
\hline $8 b$ & 5 & 4 & 3 & 2 \\
\hline $8 c$ & 7 & 6 & 5 & 4 \\
\hline $8 d$ & 7 & 7 & 6 & 4 \\
\hline $8 e$ & 4 & 3 & 2 & 1 \\
\hline $8 \mathrm{f}$ & 6 & 5 & 4 & 3 \\
\hline $9 a$ & 19 & 18 & 17 & 16 \\
\hline $9 b$ & 18 & 17 & 16 & 15 \\
\hline $10 a$ & 12 & 11 & 10 & 10 \\
\hline $10 \mathrm{~b}$ & 11 & 11 & 10 & 9 \\
\hline $11 a$ & 10 & 10 & 9 & 8 \\
\hline $11 b$ & 9 & 8 & 7 & 7 \\
\hline $11 \mathrm{c}$ & 10 & 9 & 8 & 7 \\
\hline $11 d$ & 8 & 8 & 7 & 6 \\
\hline $12 a$ & 25 & 24 & 23 & 22 \\
\hline $12 b$ & 24 & 23 & 22 & 21 \\
\hline $13 a$ & 21 & 20 & 19 & 18 \\
\hline $13 b$ & 20 & 19 & 18 & 17 \\
\hline $14 a$ & 14 & 13 & 12 & 11 \\
\hline $14 b$ & 15 & 14 & 14 & 12 \\
\hline $14 \mathrm{c}$ & 13 & 12 & 11 & 10 \\
\hline $14 d$ & 14 & 14 & 13 & 12 \\
\hline Cefotaxime sodium & 5 & 4 & 3 & 2 \\
\hline Negative control & NI & NI & NI & NI \\
\hline
\end{tabular}

DMSO was used as the negative control and as the solvent for test compounds and the reference drug. NI-No inhibition.

Table 2. A minimum inhibitory concentration of the compounds against fungi.

\begin{tabular}{ccccc}
\hline \multicolumn{5}{c}{ MIC $\left(\mu \mathrm{mol} \mathrm{mL}^{-\mathbf{1}}\right)$} \\
\hline \multirow{5}{*}{ Compounds } & $\begin{array}{c}\text { Microorganisms } \\
\text { Aspergillus } \\
\text { Niger }\end{array}$ & $\begin{array}{c}\text { Alternaria } \\
\text { Alternata }\end{array}$ & $\begin{array}{c}\text { Curvularia } \\
\text { Lunata }\end{array}$ & $\begin{array}{c}\text { Candida } \\
\text { Albicans }\end{array}$ \\
\cline { 2 - 5 } & 39 & 38 & 37 & 36 \\
1a & 38 & 36 & 34 & 33 \\
1b & 35 & 34 & 33 & 32 \\
$\mathbf{2 a}$ & 33 & 32 & 31 & 30 \\
2b & & &
\end{tabular}


Table 2. Cont.

\begin{tabular}{|c|c|c|c|c|}
\hline \multicolumn{5}{|c|}{$\operatorname{MIC}\left(\mu \mathrm{mol} \mathrm{mL}^{-1}\right)$} \\
\hline \multirow[b]{2}{*}{ Compounds } & \multicolumn{4}{|c|}{ Microorganisms } \\
\hline & $\begin{array}{c}\text { Aspergillus } \\
\text { Niger }\end{array}$ & $\begin{array}{l}\text { Alternaria } \\
\text { Alternata }\end{array}$ & $\begin{array}{l}\text { Curvularia } \\
\text { Lunata }\end{array}$ & $\begin{array}{l}\text { Candida } \\
\text { Albicans }\end{array}$ \\
\hline 3 & 42 & 40 & 39 & 38 \\
\hline $4 a$ & 31 & 30 & 29 & 28 \\
\hline $4 b$ & 30 & 29 & 27 & 26 \\
\hline $5 \mathbf{a}$ & 29 & 28 & 26 & 25 \\
\hline $5 b$ & 29 & 27 & 26 & 25 \\
\hline $6 a$ & 26 & 24 & 23 & 22 \\
\hline $6 b$ & 25 & 23 & 22 & 21 \\
\hline $7 a$ & 20 & 18 & 17 & 16 \\
\hline $7 \mathbf{b}$ & 19 & 17 & 16 & 15 \\
\hline $8 a$ & 9 & 8 & 8 & 4 \\
\hline $8 b$ & 6 & 5 & 4 & 3 \\
\hline $8 c$ & 8 & 7 & 6 & 5 \\
\hline $8 d$ & 9 & 8 & 7 & 5 \\
\hline $8 e$ & 5 & 4 & 3 & 2 \\
\hline $8 f$ & 7 & 6 & 5 & 4 \\
\hline $9 a$ & 22 & 20 & 19 & 18 \\
\hline $9 b$ & 21 & 19 & 18 & 17 \\
\hline $10 a$ & 14 & 13 & 12 & 11 \\
\hline $10 \mathrm{~b}$ & 13 & 12 & 11 & 10 \\
\hline $11 a$ & 12 & 11 & 10 & 9 \\
\hline $11 b$ & 10 & 9 & 8 & 8 \\
\hline 11c & 11 & 10 & 9 & 8 \\
\hline 11d & 9 & 9 & 8 & 7 \\
\hline $12 a$ & 28 & 26 & 25 & 24 \\
\hline $12 b$ & 27 & 25 & 24 & 23 \\
\hline $13 a$ & 24 & 22 & 21 & 20 \\
\hline $13 b$ & 23 & 21 & 20 & 19 \\
\hline $14 a$ & 16 & 15 & 14 & 13 \\
\hline $14 b$ & 18 & 16 & 15 & 15 \\
\hline $14 \mathrm{c}$ & 15 & 14 & 13 & 12 \\
\hline $14 d$ & 17 & 16 & 15 & 14 \\
\hline Nystatin & 4 & 3 & 2 & 2 \\
\hline Negative control & $\mathrm{NI}$ & NI & $\mathrm{NI}$ & NI \\
\hline
\end{tabular}

DMSO was used as the negative control and as the solvent for test compounds and the reference drug. NI-No inhibition.

\section{Experimental Section}

\subsection{General Information}

All melting points were taken on an Electrothermal IA 9100 series digital melting point apparatus (Shimadzu, Tokyo, Japan). Elemental analyses were performed on Vario EL (Elementar, Langenselbold, Germany). Microanalytical data were processed in the microanalytical center, Faculty of Science, Cairo University and National Research Centre. The IR spectra ( $\mathrm{KBr}$ disc) were recorded using a Perkin-Elmer 1650 spectrometer (Waltham, MA, USA). NMR spectra were determined using JEOL $270 \mathrm{MHz}$ and JEOL JMS-AX $500 \mathrm{MHz}$ (JEOL, Tokyo, Japan) spectrometers with $\mathrm{Me}_{4} \mathrm{Si}$ as an internal standard. Mass spectra were recorded on an EI Ms-QP 1000 EX instrument (Shimadzu, Japan) at $70 \mathrm{eV}$. Biological evaluations were done by the antimicrobial unit, Department of Chemistry of Natural and Microbial Products, National Research Centre, Egypt. All starting materials and solvents were purchased from Sigma-Aldrich (Saint Louis, MO, USA). 
3.2. General Procedure for Synthesis of 6-((1-(6-Hydroxy-(4-methoxy or 4,7-dimethoxy)-benzofuran-5-yl) ethylidene)amino)-2-thioxo-2,3-dihydropyrimidin-4(1H)-one (4a,b)

A mixture of visnaginone $\mathbf{2 a}(2.06 \mathrm{~g}, 0.01 \mathrm{~mol})$ or khellinone $\mathbf{2 b}(2.36 \mathrm{~g}, 0.01 \mathrm{~mol})$ with 6-amino2-thioxo-2,3-dihydropyrimidin-4(1H)-one $3(1.43 \mathrm{~g}, 0.01 \mathrm{~mol})$ in dimethylformamide $(40 \mathrm{~mL}) \mathrm{was}$ refluxed for 4-6 h. The solid formed was filtered off, dried, and crystallized from the proper solvent to give $4 a$ and $4 b$, respectively.

\subsection{Synthesis of 6-((1-(6-Hydroxy-4-methoxybenzofuran-5-yl)ethylidene)amino)-2-thioxo-2,3-dihydro} pyrimidin-4(1H)-one (4a)

The compound was obtained from the reaction of visnaginone (2a) with 6-amino-thiouracil (3) as yellow crystals, crystallized from methanol in $90 \%$ yield, m.p. $231^{\circ} \mathrm{C}$. IR $\left(v, \mathrm{~cm}^{-1}\right) \mathrm{KBr}: 3370$ (brs, 2NH), 3030 (CH-aryl), 2960 (CH-aliph), 1685 (CO, amide), 1630 (C=N). ${ }^{1} \mathrm{H}-\mathrm{NMR}$ (DMSO- $\left.d_{6}, \mathrm{ppm}\right) \delta$ $2.38\left(\mathrm{~s}, 3 \mathrm{H}, \mathrm{CH}_{3}\right), 3.92\left(\mathrm{~s}, 3 \mathrm{H}, \mathrm{OCH}_{3}\right), 6.79(\mathrm{~d}, 1 \mathrm{H}, \mathrm{J}=2.35 \mathrm{~Hz}$, furan), 7.80 (d, 1H, J = 2.38 Hz, furan), 7.92 (s, 1H, phenyl), 7.94 (s, 1H, pyrimidine), 10.80, 11.19 (br, 2H, 2NH, $\mathrm{D}_{2} \mathrm{O}$ exchangeable), 16.11 (br, $1 \mathrm{H}, \mathrm{OH}, \mathrm{D}_{2} \mathrm{O}$ exchangeable); ${ }^{13} \mathrm{C}-\mathrm{NMR}$ (DMSO-d 6$) \delta 21.3\left(1 \mathrm{C}, \mathrm{CH}_{3}\right), 61.7\left(1 \mathrm{C}, \mathrm{OCH}_{3}\right), 93.1(1 \mathrm{C}, \mathrm{CH}$, pyrimidine), 98.9 (1C, CH, phenyl), 100.5, 104.5, 108.9, 146.6, 146.9, 154.5, 160.9, 163.2, (8C, Ar-C), 164.1 (1C, C=N-ph), 168.1(1C, C=O), $173.5(1 \mathrm{C}, \mathrm{C}=\mathrm{S}) ; \mathrm{MS}(70 \mathrm{eV}, \%) \mathrm{m} / \mathrm{z} 331\left(\mathrm{M}^{+}, 100 \%\right)$; Anal. Calc. (Found) for $\mathrm{C}_{15} \mathrm{H}_{13} \mathrm{~N}_{3} \mathrm{O}_{4} \mathrm{~S}$ (331.35): $\mathrm{C}, 54.37$ (54.30); H, 3.95 (3.91); N, 12.68(12.60).

\subsection{Synthesis of 6-((1-(6-Hydroxy-4,7-dimethoxybenzofuran-5-yl)ethylidene)amino)-2-thioxo-2,3-dihydro pyrimidin-4(1H)-one (4b)}

The compound was obtained from the reaction of khellinone (2b) with 6-amino-thiouracil (3) as yellowish crystals, crystallized from dioxane in $84 \%$ yield, m.p. $211^{\circ} \mathrm{C}$. IR $\left(v, \mathrm{~cm}^{-1}\right) \mathrm{KBr}$ : 3371 (brs, 2NH), 3032 (CH-aryl), 2965 (CH-aliph), 1682 (CO, amide), 1631 (C=N). ${ }^{1} \mathrm{H}-\mathrm{NMR}$ (DMSO- $\left.d_{6}, \mathrm{ppm}\right)$ $\delta 2.39\left(\mathrm{~s}, 3 \mathrm{H}, \mathrm{CH}_{3}\right), 3.94\left(\mathrm{~s}, 6 \mathrm{H}, 2 \mathrm{OCH}_{3}\right), 6.80(\mathrm{~d}, 1 \mathrm{H}, J=2.34 \mathrm{~Hz}$, furan), $7.83(\mathrm{~d}, 1 \mathrm{H}, J=2.37 \mathrm{~Hz}$, furan), 7.95 (s, 1H, pyrimidine), 10.81,11.20 (br, 2H, 2NH, $\mathrm{D}_{2} \mathrm{O}$ exchangeable), $16.12\left(\mathrm{br}, 1 \mathrm{H}, \mathrm{OH}, \mathrm{D}_{2} \mathrm{O}\right.$ exchangeable); ${ }^{13} \mathrm{C}-\mathrm{NMR}\left(\mathrm{DMSO}-d_{6}\right) \delta 21.4\left(1 \mathrm{C}, \mathrm{CH}_{3}\right), 61.6\left(2 \mathrm{C}, \mathrm{OCH}_{3}\right), 93.2(1 \mathrm{C}, \mathrm{CH}$, pyrimidine), 100.5, 104.7, 109.9, 129.3, 146.2, 147.1, 153.8, 158.9, 164.7, (9C, Ar-C), 165.2 (1C, C=N-ph), 167.9 (1C, $\mathrm{C}=\mathrm{O}), 173.8(1 \mathrm{C}, \mathrm{C}=\mathrm{S})$; MS (70 eV, \%) m/z $361\left(\mathrm{M}^{+}, 100 \%\right)$; Anal. Calc. (Found) for $\mathrm{C}_{16} \mathrm{H}_{15} \mathrm{~N}_{3} \mathrm{O}_{5} \mathrm{~S}$ (361.37): C, 53.18 (53.23); H, 4.18 (4.13); N, 11.63 (11.68).

\subsection{General Procedure for Synthesis of (7-Methoxy or 7,11-dimethoxy)-6-methyl-1-thioxo-1,2-dihydro-} 3 H-furo[3,2-g]pyrimido [1,6-a]quinazolin-3-one (5a,b)

Method A: A mix of visnaginone $2 \mathbf{a}(2.06 \mathrm{~g}, 0.01 \mathrm{~mol})$ or khellinone $2 \mathbf{b}(2.36 \mathrm{~g}, 0.01 \mathrm{~mol})$ with 6-amino-2-thiouracil (3) in DMF (40 mL) was refluxed for 14-16 h. The product precipitated was filtered off and washed with $100 \mathrm{~mL}$ water, dried and crystallized from the proper solvent to give 5a and $5 \mathbf{b}$, respectively. Method B: A refluxing of $4 \mathbf{a}(3.31 \mathrm{~g}, 0.01 \mathrm{~mol})$ or $4 \mathbf{b}(3.61 \mathrm{~g}, 0.01 \mathrm{~mol})$ in DMF $(40 \mathrm{~mL})$ was refluxed for $8-10 \mathrm{~h}$ under control (TLC). The final precipitated was filtered off and washed with $50 \mathrm{~mL}$ ethanol, dried, and crystallized from the proper solvent to give $\mathbf{5 a}$ and $\mathbf{5 b}$, respectively.

\subsection{Synthesis of 7-Methoxy-6-methyl-1-thioxo-1,2-dihydro-3H-furo[3,2-g]pyrimido[1,6-a]quinazolin- 3-one (5a)}

The compound was obtained from the reaction of visnaginone (2a) with 6-aminothiouracil (3) or compound (4a) in dimethylformamide, as yellowish crystals, crystallized from benzene in $85 \%$ yield, m.p. $271{ }^{\circ} \mathrm{C}$. IR (v, cm ${ }^{-1}$ ) KBr: 3350 (brs, NH), 3028 (CH-aryl), 2955 (CH-aliph), 1680 (CO, amide), $1635(\mathrm{C}=\mathrm{N}) .{ }^{1} \mathrm{H}-\mathrm{NMR}\left(\mathrm{DMSO}-d_{6}, \mathrm{ppm}\right) \delta 2.32\left(\mathrm{~s}, 3 \mathrm{H}, \mathrm{CH}_{3}\right), 3.92\left(\mathrm{~s}, 3 \mathrm{H}, \mathrm{OCH}_{3}\right), 6.75(\mathrm{~d}, 1 \mathrm{H}$, $J=2.33 \mathrm{~Hz}$, furan), 7.33 (s, 1H, pyrimidine), 7.80 (d, 1H, $J=2.36 \mathrm{~Hz}$, furan), 8.29 (s, 1H, phenyl), 11.20 (br, 1H, NH, D $\mathrm{O}$ exchangeable); ${ }^{13} \mathrm{C}-\mathrm{NMR}$ (DMSO-d $) \delta 24.5\left(1 \mathrm{C}, \mathrm{CH}_{3}\right), 61.8\left(1 \mathrm{C}, \mathrm{OCH}_{3}\right), 79.7(1 \mathrm{C}$, $\mathrm{CH}$, pyrimidine), 99.1 (1C, CH, phenyl), 104.9, 108.9,116.6, 140.9, 146.6, 148.9, 160.1, 161.2, 164.1 (9C, 
Ar-C), 168.2 (1C, C=O), $173.6(1 \mathrm{C}, \mathrm{C}=\mathrm{S})$; $\mathrm{MS}(70 \mathrm{eV}, \%) \mathrm{m} / \mathrm{z} 313\left(\mathrm{M}^{+}, 100 \%\right)$; Anal. Calc. (Found) for $\mathrm{C}_{15} \mathrm{H}_{11} \mathrm{~N}_{3} \mathrm{O}_{3} \mathrm{~S}$ (313.33): $\mathrm{C}, 57.50$ (57.58); $\mathrm{H}, 3.54$ (3.59); N, 13.41(13.50).

\subsection{Synthesis of 7,11-Dimethoxy-6-methyl-1-thioxo-1,2-dihydro-3H-furo[3,2-g]pyrimido[1,6-a]} quinazolin-3-one (5b)

The compound was obtained from the reaction of khellinone (2b) with 6-amino-thiouracil (3) or compound $(\mathbf{4 b})$ in dimethylformamide, as yellow crystals, crystallized from toluene in $82 \%$ yield, m.p. $251{ }^{\circ} \mathrm{C}$. IR ( $\left.v, \mathrm{~cm}^{-1}\right) \mathrm{KBr}: 3352$ (brs, NH), 3029 (CH-aryl), 2957 (CH-aliph), 1681 (CO, amide), 1633 $(\mathrm{C}=\mathrm{N}) .{ }^{1} \mathrm{H}-\mathrm{NMR}\left(\mathrm{DMSO}-d_{6}, \mathrm{ppm}\right) \delta 2.35\left(\mathrm{~s}, 3 \mathrm{H}, \mathrm{CH}_{3}\right), 3.95\left(\mathrm{~s}, 6 \mathrm{H}, 2 \mathrm{OCH}_{3}\right), 6.77(\mathrm{~d}, 1 \mathrm{H}, J=2.31 \mathrm{~Hz}$, furan), $7.30\left(\mathrm{~s}, 1 \mathrm{H}\right.$, pyrimidine), $7.82\left(\mathrm{~d}, 1 \mathrm{H}, J=2.37 \mathrm{~Hz}\right.$, furan), $11.25\left(\mathrm{br}, 1 \mathrm{H}, \mathrm{NH}, \mathrm{D}_{2} \mathrm{O}\right.$ exchangeable); ${ }^{13} \mathrm{C}-\mathrm{NMR}\left(\mathrm{DMSO}-d_{6}\right) \delta 24.6\left(1 \mathrm{C}, \mathrm{CH}_{3}\right), 61.9\left(2 \mathrm{C}, 2 \mathrm{OCH}_{3}\right), 80.2(1 \mathrm{C}, \mathrm{CH}$, pyrimidine), 105.1, 109.2, 115.9, 125.8, 137.5, 142.8, 146.5, 149.2, 161.7, 164.8 (10C, Ar-C), 168.4 (1C, C=O), 173.8 (1C, C=S); MS (70 eV, \%) $\mathrm{m} / \mathrm{z} 343\left(\mathrm{M}^{+}, 100 \%\right)$; Anal. Calc. (Found) for $\mathrm{C}_{16} \mathrm{H}_{13} \mathrm{~N}_{3} \mathrm{O}_{4} \mathrm{~S}$ (343.36): $\mathrm{C}, 55.97$ (55.90); $\mathrm{H}, 3.82$ (3.75); $\mathrm{N}$, $12.24(12.29)$.

3.8. General Procedure for Synthesis of 2-(((7-Methoxy or 7,11-dimethoxy)-6-methyl-3-oxo-3H-furo[3,2-g] pyrimido[1,6-a]quinazolin-1-yl)thio) Acetic Acid (6a,b)

A mixture from $5 \mathbf{a}(3.13 \mathrm{~g}, 0.01 \mathrm{~mol})$ or $5 \mathbf{b}(3.43 \mathrm{~g}, 0.01 \mathrm{~mol})$, chloroacetic acid $(0.94 \mathrm{~g}, 0.01 \mathrm{~mol})$ and $(0.02 \mathrm{~mol})$ of anhydrous sodium acetate was stirred under reflux in $40 \mathrm{~mL}$ of glacial acetic acid and $20 \mathrm{~mL}$ of acetic anhydride for $3-5 \mathrm{~h}$. The reaction mixture was cooled and poured into cold water $(100 \mathrm{~mL})$. The deposited precipitate was filtered off, and crystallized from appropriate solvent to produce $6 \mathbf{a}$ and $6 \mathrm{~b}$, respectively.

\subsection{Synthesis of 2-((7-Methoxy-6-methyl-3-oxo-3H-furo[3,2-g]pyrimido[1,6-a]quinazolin-1-yl)thio)} Acetic Acid (6a)

The compound was obtained from the reaction of (5a) with chloroacetic acid, as brownish crystals, crystallized from hexane in 88\% yield, m.p. $294{ }^{\circ} \mathrm{C}$. IR ( $\left.v, \mathrm{~cm}^{-1}\right) \mathrm{KBr}: 3340$ (brs, OH), 3025 (CH-aryl), 2950 (CH-aliph), 1745 (CO, acid), 1684 (CO, amide), 1631 (C=N). ${ }^{1} \mathrm{H}-\mathrm{NMR}$ (DMSO- $\left.d_{6}, \mathrm{ppm}\right) \delta 2.30$ (s, $\left.3 \mathrm{H}, \mathrm{CH}_{3}\right), 3.91\left(\mathrm{~s}, 3 \mathrm{H}, \mathrm{OCH}_{3}\right), 4.27\left(\mathrm{~s}, 2 \mathrm{H}, \mathrm{CH}_{2}\right), 6.77(\mathrm{~d}, 1 \mathrm{H}, \mathrm{J}=2.32 \mathrm{~Hz}$, furan), $7.35(\mathrm{~s}, 1 \mathrm{H}$, pyrimidine), $7.78\left(\mathrm{~d}, 1 \mathrm{H}, J=2.35 \mathrm{~Hz}\right.$, furan), $7.90\left(\mathrm{~s}, 1 \mathrm{H}\right.$, phenyl), $13.70\left(\mathrm{br}, 1 \mathrm{H}, \mathrm{OH}, \mathrm{D}_{2} \mathrm{O}\right.$ exchangeable); ${ }^{13} \mathrm{C}-\mathrm{NMR}$ (DMSO-d $\left.d_{6}\right) \delta 23.6\left(1 \mathrm{C}, \mathrm{CH}_{3}\right), 32.8\left(1 \mathrm{C}, \mathrm{CH}_{2}\right), 61.7\left(1 \mathrm{C}, \mathrm{OCH}_{3}\right), 97.2(1 \mathrm{C}, \mathrm{CH}$, phenyl), $100.6(1 \mathrm{C}, \mathrm{CH}$, pyrimidine), 103.4, 105.3, 108.5, 143.7, 146.5, 152.2,158.4, 160.6, 163.9,164.8 (10C, Ar-C), 169.1, 172.8 (2C, $2 \mathrm{C}=\mathrm{O})$; $\mathrm{MS}(70 \mathrm{eV}, \%) \mathrm{m} / z 371\left(\mathrm{M}^{+}, 100 \%\right.$ ); Anal. Calc. (Found) for $\mathrm{C}_{17} \mathrm{H}_{13} \mathrm{~N}_{3} \mathrm{O}_{5} \mathrm{~S}$ (371.37): $\mathrm{C}, 54.98$ (54.91); H, 3.53 (3.58); N, 11.32(11.40).

\subsection{Synthesis of 2-((7,11-Dimethoxy-6-methyl-3-oxo-3H-furo[3,2-g]pyrimido[1,6-a]quinazolin-1-yl)thio)} Acetic Acid (6b)

The compound was obtained from the reaction of $(\mathbf{5 b})$ with chloroacetic acid, as yellowish crystals, crystallized from ethanol in $86 \%$ yield, m.p. $283^{\circ} \mathrm{C}$. IR $\left(v, \mathrm{~cm}^{-1}\right) \mathrm{KBr}: 3345$ (brs, OH), 3027 (CH-aryl), 2952 (CH-aliph), 1748 (CO, acid), 1681 (CO, amide), 1632 (C=N). ${ }^{1} \mathrm{H}-\mathrm{NMR}$ (DMSO-d $d_{6}, \mathrm{ppm}$ ) $\delta 2.31$ (s, $\left.3 \mathrm{H}, \mathrm{CH}_{3}\right), 3.95\left(\mathrm{~s}, 6 \mathrm{H}, 2 \mathrm{OCH}_{3}\right), 4.25\left(\mathrm{~s}, 2 \mathrm{H}, \mathrm{CH}_{2}\right), 6.79(\mathrm{~d}, 1 \mathrm{H}, \mathrm{J}=2.35 \mathrm{~Hz}$, furan), $7.38(\mathrm{~s}, 1 \mathrm{H}$, pyrimidine), $7.80\left(\mathrm{~d}, 1 \mathrm{H}, J=2.35 \mathrm{~Hz}\right.$, furan), 13.75 (br, $1 \mathrm{H}, \mathrm{OH}, \mathrm{D}_{2} \mathrm{O}$ exchangeable); ${ }^{13} \mathrm{C}-\mathrm{NMR}$ (DMSO- $\left.d_{6}\right) \delta 23.4(1 \mathrm{C}$, $\left.\mathrm{CH}_{3}\right), 32.6\left(1 \mathrm{C}, \mathrm{CH}_{2}\right), 61.3\left(2 \mathrm{C}, 2 \mathrm{OCH}_{3}\right), 100.1$ (1C, $\mathrm{CH}$, pyrimidine), 101.2, 105.4, 108.8, 124.1, 130.1, 146.2, 146.7, 150.5, 160.1, 164.4, 166.9 (11C, Ar-C), 169.5, 172.9 (2C, 2C=O); MS (70 eV, \%) m/z $401\left(\mathrm{M}^{+}\right.$, 100\%); Anal. Calc. (Found) for $\mathrm{C}_{18} \mathrm{H}_{15} \mathrm{~N}_{3} \mathrm{O}_{6} \mathrm{~S}$ (401.39): C, 53.86 (53.80); H, 3.77 (3.71); N, 10.47 (10.42).

3.11. General Procedure for Synthesis of (9-Methoxy or 9,13-dimethoxy)-8-methyl-2-hydro-5H,14aHfuro[3,2-g]thiazolo[2',3':2,3] pyrimido[1,6-a]quinazoline-3,5-dione (7a, b)

Method A: A mixture from $5 \mathbf{a}(3.13 \mathrm{~g}, 0.01 \mathrm{~mol})$ or $5 \mathbf{b}(3.43 \mathrm{~g}, 0.01 \mathrm{~mol})$, chloroacetic acid $(0.94 \mathrm{~g}$, $0.01 \mathrm{~mol})$ and $(0.02 \mathrm{~mol})$ of anhydrous sodium acetate was stirred under reflux in $40 \mathrm{~mL}$ of glacial acetic 
acid and $20 \mathrm{~mL}$ of acetic anhydride on a water bath $\left(60-70^{\circ} \mathrm{C}\right)$ for $13-15 \mathrm{~h}$ (TLC). The reaction mixture was allowed to cool to room temperature and poured into water $(100 \mathrm{~mL})$. The solid precipitate was filtered off, and crystallized from appropriate solvent to produced $\mathbf{7 a}$ and $\mathbf{7 b}$ in good yields, respectively. Method B: A mixture of $\mathbf{6 a}(3.71 \mathrm{~g}, 0.01 \mathrm{~mol})$ or $\mathbf{6 b}(4.01 \mathrm{~g}, 0.01 \mathrm{~mol})$ in dimethylformamide $(35 \mathrm{~mL})$ was refluxed for $6-8 \mathrm{~h}$ with (TLC). The final product was filtered off, dried and crystallized from the proper solvent to give $7 \mathbf{a}$ and $7 \mathbf{b}$, respectively.

\subsection{Synthesis of 9-Methoxy-8-methyl-2-hydro-5H,14aH-furo[3,2-g]thiazolo[ $\left[2^{\prime}, 3^{\prime}: 2,3\right]$ pyrimido[1,6-a] quinazoline-3,5-dione (7a)}

The compound was obtained from the reaction of (5a) with chloroacetic acid or compound (6a) in DMF, as white crystals, crystallized from dioxane in $82 \%$ yield, m.p. $338^{\circ} \mathrm{C}$. IR $\left(v, \mathrm{~cm}^{-1}\right) \mathrm{KBr}: 3035$ (CH-aryl), 2962 (CH-aliph), 1688, 1680 (2CO, amide), 1635 (C=N). ${ }^{1} \mathrm{H}-\mathrm{NMR}$ (DMSO- $\left.d_{6}, \mathrm{ppm}\right) \delta 2.37$ (s, $\left.3 \mathrm{H}, \mathrm{CH}_{3}\right), 3.93\left(\mathrm{~s}, 3 \mathrm{H}, \mathrm{OCH}_{3}\right), 4.21\left(\mathrm{~s}, 2 \mathrm{H}, \mathrm{CH}_{2}\right), 5.57(\mathrm{~s}, 1 \mathrm{H}, \mathrm{CH}$, thiazole), $6.78(\mathrm{~d}, 1 \mathrm{H}, J=2.34 \mathrm{~Hz}$, furan), $7.38\left(\mathrm{~s}, 1 \mathrm{H}\right.$, pyrimidine), $7.75\left(\mathrm{~d}, 1 \mathrm{H}, J=2.32 \mathrm{~Hz}\right.$, furan), $7.88\left(\mathrm{~s}, 1 \mathrm{H}\right.$, phenyl); ${ }^{13} \mathrm{C}-\mathrm{NMR}$ (DMSO- $\left.d_{6}\right) \delta$ $23.3\left(1 \mathrm{C}, \mathrm{CH}_{3}\right), 32.6\left(1 \mathrm{C}, \mathrm{CH}_{2}\right), 61.8\left(1 \mathrm{C}, \mathrm{OCH}_{3}\right), 76.1(1 \mathrm{C}, \mathrm{CH}$, thiazole), 94.5 (1C, $\mathrm{CH}$, phenyl), 98.8 (1C, CH, pyrimidine), 101.2, 105.5, 107.9, 142.2, 146.4, 153.5, 159.7, 160.4, 164.5 (9C, Ar-C), 166.4, 170.1 $(2 \mathrm{C}, 2 \mathrm{C}=\mathrm{O})$; $\mathrm{MS}(70 \mathrm{eV}, \%) \mathrm{m} / \mathrm{z} 355\left(\mathrm{M}^{+}, 90 \%\right)$; Anal. Calc. (Found) for $\mathrm{C}_{17} \mathrm{H}_{13} \mathrm{~N}_{3} \mathrm{O}_{4} \mathrm{~S}$ (355.37): $\mathrm{C}, 57.46$ (57.40); H, 3.69 (3.62); N, 11.82 (11.88).

\subsection{Synthesis of 9,13-Dimethoxy-8-methyl-2-hydro-5H,14aH-furo[3,2-g]thiazolo[2',3':2,3]pyrimido[1,6-a] quinazoline-3,5-dione ( $7 \mathbf{b}$ )}

The compound was obtained from the reaction of $(\mathbf{5 b})$ with chloroacetic acid or compound $(\mathbf{6 b})$ in DMF, as yellowish crystals, crystallized from methanol in $80 \%$ yield, m.p. $303^{\circ} \mathrm{C}$. IR $\left(v, \mathrm{~cm}^{-1}\right) \mathrm{KBr}$ : 3038 (CH-aryl), 2966 (CH-aliph), 1685, 1682 (2CO, amide), 1632 (C=N). ${ }^{1} \mathrm{H}-\mathrm{NMR}$ (DMSO- $\left.d_{6}, \mathrm{ppm}\right) \delta$ $2.33\left(\mathrm{~s}, 3 \mathrm{H}, \mathrm{CH}_{3}\right), 3.97\left(\mathrm{~s}, 6 \mathrm{H}, 2 \mathrm{OCH}_{3}\right), 4.24\left(\mathrm{~s}, 2 \mathrm{H}, \mathrm{CH}_{2}\right), 5.62(\mathrm{~s}, 1 \mathrm{H}, \mathrm{CH}$, thiazole), $6.74(\mathrm{~d}, 1 \mathrm{H}, J=2.33$ $\mathrm{Hz}$, furan), $7.39\left(\mathrm{~s}, 1 \mathrm{H}\right.$, pyrimidine), $7.77\left(\mathrm{~d}, 1 \mathrm{H}, J=2.31 \mathrm{~Hz}\right.$, furan); ${ }^{13} \mathrm{C}-\mathrm{NMR}$ (DMSO- $\left.d_{6}\right) \delta 23.1(1 \mathrm{C}$, $\left.\mathrm{CH}_{3}\right), 32.3\left(1 \mathrm{C}, \mathrm{CH}_{2}\right), 61.7\left(2 \mathrm{C}, 2 \mathrm{OCH}_{3}\right), 76.4(1 \mathrm{C}, \mathrm{CH}$, thiazole), 97.5 (1C, $\mathrm{CH}$, pyrimidine), 101.4, 105.6, 108.2, 123.6, 127.8, 146.3, 146.8, 152.9, 160.1, 164.7 (10C, Ar-C), 166.2, 170.5 (2C, 2C=O); MS (70 eV, \%) $m / z 385\left(\mathrm{M}^{+}, 95 \%\right)$; Anal. Calc. (Found) for $\mathrm{C}_{18} \mathrm{H}_{15} \mathrm{~N}_{3} \mathrm{O}_{5} \mathrm{~S}$ (385.39): C, 56.10 (56.18); $\mathrm{H}, 3.92$ (3.85); $\mathrm{N}$, $10.90(10.98)$.

3.14. General Procedure for Synthesis of 2-(Substituted-benzylidene)-9,(substituted)-methoxy-8-methyl-2hydro-5H,14aH-furo[3,2-g] thiazolo[2',3':2,3]pyrimido[1,6-a]quinazoline-3,5-dione (8a-f)

Method A: One pot synthesis: A mixture from $5 \mathbf{a}(3.13 \mathrm{~g}, 0.01 \mathrm{~mol})$ or $5 \mathbf{b}(3.43 \mathrm{~g}, 0.01 \mathrm{~mol})$, chloroacetic acid $(0.94 \mathrm{~g}, 0.01 \mathrm{~mol})$, the appropriate aromatic aldehyde $(10 \mathrm{mmol})$ and $(0.02 \mathrm{~mol})$ of anhydrous sodium acetate was stirred under reflux in $40 \mathrm{~mL}$ of glacial acetic acid and $20 \mathrm{~mL}$ of acetic anhydride for $24-26 \mathrm{~h}$. The reaction mixture was cooled and poured into ice water. The deposited precipitate was filtered off, and crystallized from appropriate solvent to give (8a-f).

Method B: A mixture of compound $7 \mathbf{a}(3.55 \mathrm{~g}, 10 \mathrm{mmol})$ or $7 \mathbf{b}(3.85 \mathrm{~g}, 10 \mathrm{mmol})$ and the appropriate aromatic aldehyde $(10 \mathrm{mmol})$ in dioxane $(40 \mathrm{~mL})$ containing a catalyst amount of piperidine $(0.5 \mathrm{~mL})$ was stirred and heated under reflux for 10-12 h (TLC control). The reaction mixture was cooled, the formed precipitate filtered off, dried and recrystallized from the appropriate solvent to afford (8a-f).

3.15. Synthesis of 2-Benzylidene-9-methoxy-8-methyl-2-hydro-5H,14aH-furo[3,2-g]thiazolo[2',3':2,3] pyrimido[1,6-a]quinazoline-3,5-dione (8a)

The compound was obtained from the reaction of (5a) and benzaldehyde (1.06 g, $10 \mathrm{mmol})$ with chloroacetic acid or compound (7a) with benzaldehyde, as yellowish crystals, crystallized from dimethylformamide in $75 \%$ yield, m.p. $>350^{\circ} \mathrm{C}$. IR $\left(v, \mathrm{~cm}^{-1}\right) \mathrm{KBr}: 3050$ (CH-aryl), 2945 (CH-aliph), 1685, 1672 (2CO, amide), 1630 (C=N). ${ }^{1} \mathrm{H}-\mathrm{NMR}$ (DMSO- $\left.d_{6}, \mathrm{ppm}\right) \delta 2.30\left(\mathrm{~s}, 3 \mathrm{H}, \mathrm{CH}_{3}\right), 3.91\left(\mathrm{~s}, 3 \mathrm{H}, \mathrm{OCH}_{3}\right)$, $5.59(\mathrm{~s}, 1 \mathrm{H}, \mathrm{CH}$, thiazole), $6.75(\mathrm{~d}, 1 \mathrm{H}, J=2.31 \mathrm{~Hz}$, furan), $7.32(\mathrm{~s}, 1 \mathrm{H}$, pyrimidine), $7.40-7.67(\mathrm{~m}, 5 \mathrm{H}$, 
phenyl), $7.72\left(\mathrm{~d}, 1 \mathrm{H}, J=2.30 \mathrm{~Hz}\right.$, furan), $7.85\left(\mathrm{~s}, 1 \mathrm{H}\right.$, phenyl), $8.05(\mathrm{~s}, 1 \mathrm{H}, \mathrm{CH}) ;{ }^{13} \mathrm{C}-\mathrm{NMR}\left(\mathrm{DMSO}-d_{6}\right)$ $\delta 23.1\left(1 \mathrm{C}, \mathrm{CH}_{3}\right), 61.6\left(1 \mathrm{C}, \mathrm{OCH}_{3}\right), 77.5(1 \mathrm{C}, \mathrm{CH}$, thiazole), $90.8(1 \mathrm{C}, \mathrm{CH}$, phenyl), $92.9(1 \mathrm{C}, \mathrm{CH}$, pyrimidine), 100.7, 104.8, 105.6, (3C, benzofurane), 121.9 (1C, CH), 127.2, 128.1, 128.7, 133.9 (6C, phenyl), 137.5 (1C, thiazole), 140.3, 146.4, 153.8, 160.1, 160.4, 164.2 (6C, pyrimidobenzo furane), 165.6, $168.4(2 \mathrm{C}, 2 \mathrm{C}=\mathrm{O})$; $\mathrm{MS}(70 \mathrm{eV}, \%) \mathrm{m} / z 443\left(\mathrm{M}^{+}, 88 \%\right)$; Anal. Calc. (Found) for $\mathrm{C}_{24} \mathrm{H}_{17} \mathrm{~N}_{3} \mathrm{O}_{4} \mathrm{~S}$ (443.48): $\mathrm{C}$, 65.00 (65.10); H, 3.86 (3.80); N, 9.48 (9.41).

3.16. Synthesis of 2-(4-Chlorobenzylidene)-9-methoxy-8-methyl-2-hydro-5H,14aH-furo[3,2-g]thiazolo $\left[2^{\prime}, 3^{\prime}: 2,3\right]$ pyrimido[1,6-a]quinazoline-3,5-dione (8b)

The compound was obtained from the reaction of (5a) and 4-chlorobenzaldehyde (1.40 g, $10 \mathrm{mmol})$ with chloroacetic acid or compound (7a) with 4-chloro-benzaldehyde, as yellow crystals, crystallized from dioxane in $80 \%$ yield, m.p. $>350{ }^{\circ} \mathrm{C}$. IR $\left(v, \mathrm{~cm}^{-1}\right) \mathrm{KBr}: 3052$ (CH-aryl), 2948 (CH-aliph), 1687, $1674\left(2 \mathrm{CO}\right.$, amide), $1631(\mathrm{C}=\mathrm{N}) .{ }^{1} \mathrm{H}-\mathrm{NMR}\left(\mathrm{DMSO}_{-} d_{6}, \mathrm{ppm}\right) \delta 2.31\left(\mathrm{~s}, 3 \mathrm{H}, \mathrm{CH}_{3}\right), 3.90\left(\mathrm{~s}, 3 \mathrm{H}, \mathrm{OCH}_{3}\right)$, $5.60(\mathrm{~s}, 1 \mathrm{H}, \mathrm{CH}$, thiazole), $6.74(\mathrm{~d}, 1 \mathrm{H}, J=2.32 \mathrm{~Hz}$, furan), $7.33(\mathrm{~s}, 1 \mathrm{H}$, pyrimidine $), 7.45-7.50(\mathrm{dd}, 2 \mathrm{H}$, $J=7.60,7.64 \mathrm{~Hz}$, 4-chlorophenyl), 7.55-7.60 (dd, 2H, $J=7.62,7.66 \mathrm{~Hz}, 4$-chloro phenyl),7.70 (d, $1 \mathrm{H}$, $J=2.34 \mathrm{~Hz}$, furan), $7.83\left(\mathrm{~s}, 1 \mathrm{H}\right.$, phenyl), $8.08(\mathrm{~s}, 1 \mathrm{H}, \mathrm{CH}) ;{ }^{13} \mathrm{C}-\mathrm{NMR}\left(\mathrm{DMSO}-d_{6}\right) \delta 23.2\left(1 \mathrm{C}, \mathrm{CH}_{3}\right), 61.4$ (1C, $\left.\mathrm{OCH}_{3}\right), 77.8$ (1C, $\mathrm{CH}$, thiazole), 90.2 (1C, $\mathrm{CH}$, phenyl), 92.1 (1C, $\mathrm{CH}$, pyrimidine), 100.3, 104.9, 105.5, (3C, benzofurane), 122.1 (1C, CH), 128.4, 129.2, 132.5, 132.8 (6C, 4-chlorophenyl), 137.9 (1C, thiazole), 140.6, 146.3,153.5, 160.3, 160.7, 164.3 (6C, pyrimidobenzo furane), 165.8, $168.7(2 \mathrm{C}, 2 \mathrm{C}=\mathrm{O})$; MS (70 eV, \%) m/z $477\left(\mathrm{M}^{+}, 90 \%\right)$; Anal. Calc. (Found) for $\mathrm{C}_{24} \mathrm{H}_{16} \mathrm{ClN}_{3} \mathrm{O}_{4} \mathrm{~S}$ (477.92): $\mathrm{C}, 60.32$ (60.39); $\mathrm{H}$, 3.37 (3.44); $\mathrm{N}, 8.79$ (8.85).

3.17. Synthesis of 9-Methoxy-2-(4-methoxybenzylidene)-8-methyl-2-hydro-5H,14aH-furo[3,2-g]thiazolo $\left[2^{\prime}, 3^{\prime}: 2,3\right]$ pyrimido $[1,6-$ a]quinazoline-3,5-dione (8c)

The compound was obtained from the reaction of (5a) and 4-methoxy-benzaldehyde (1.36 $\mathrm{g}$, $10 \mathrm{mmol}$ ) with chloroacetic acid or compound (7a) with 4-methoxybenzaldehyde, as brownish crystals, crystallized from ethanol in $78 \%$ yield, m.p. $>350{ }^{\circ} \mathrm{C}$. IR $\left(v, \mathrm{~cm}^{-1}\right) \mathrm{KBr}: 3054(\mathrm{CH}$-aryl), 2944 (CH-aliph), 1683, 1671 (2CO, amide), $1636(\mathrm{C}=\mathrm{N}) .{ }^{1} \mathrm{H}-\mathrm{NMR}$ (DMSO-d $\left.d_{6}, \mathrm{ppm}\right) \delta 2.29\left(\mathrm{~s}, 3 \mathrm{H}, \mathrm{CH}_{3}\right), 3.92$ $\left(\mathrm{s}, 3 \mathrm{H}, \mathrm{OCH}_{3}\right), 4.10\left(\mathrm{~s}, 3 \mathrm{H}, \mathrm{OCH}_{3}\right), 5.59(\mathrm{~s}, 1 \mathrm{H}, \mathrm{CH}$, thiazole $), 6.71(\mathrm{~d}, 1 \mathrm{H}, J=2.32 \mathrm{~Hz}$, furan), $7.28(\mathrm{~s}$, $1 \mathrm{H}$, pyrimidine), $7.48-7.53(\mathrm{dd}, 2 \mathrm{H}, J=7.61,7.65 \mathrm{~Hz}, 4$-methoxyphenyl), 7.58-7.63 (dd, $2 \mathrm{H}, J=7.63$, $7.67 \mathrm{~Hz}$, 4-methoxyphenyl), $7.69(\mathrm{~d}, 1 \mathrm{H}, J=2.37 \mathrm{~Hz}$, furan), $7.86(\mathrm{~s}, 1 \mathrm{H}$, phenyl), $8.06(\mathrm{~s}, 1 \mathrm{H}, \mathrm{CH})$; ${ }^{13} \mathrm{C}-\mathrm{NMR}\left(\mathrm{DMSO}-d_{6}\right) \delta 23.4\left(1 \mathrm{C}, \mathrm{CH}_{3}\right), 58.5\left(1 \mathrm{C}, \mathrm{OCH}_{3}\right), 61.7\left(1 \mathrm{C}, \mathrm{OCH}_{3}\right), 77.2(1 \mathrm{C}, \mathrm{CH}$, thiazole), 90.5 (1C, $\mathrm{CH}$, phenyl), 92.4 (1C, CH, pyrimidine), 100.4, 105.1, 105.5, (3C, benzofurane), $122.6(1 \mathrm{C}, \mathrm{CH})$, 123.1, 127.7, 130.1, 155.5 (6C, 4-methoxyphenyl), 137.4 (1C, thiazole), 140.8, 146.2,153.6, 160.1, 160.4, 164.3 (6C, pyrimidobenzofurane), 165.1, 168.6 (2C, 2C=O); $\mathrm{MS}(70 \mathrm{eV}, \%) \mathrm{m} / z 473\left(\mathrm{M}^{+}, 87 \%\right)$; Anal. Calc. (Found) for $\mathrm{C}_{25} \mathrm{H}_{19} \mathrm{~N}_{3} \mathrm{O}_{5} \mathrm{~S}$ (473.50): C, 63.42 (63.49); $\mathrm{H}, 4.04$ (4.12); N, 8.87 (8.95).

\subsection{Synthesis of 2-Benzylidene-9,13-dimethoxy-8-methyl-2-hydro-5H,14aH-furo[3,2-g]thiazolo} $\left[2^{\prime}, 3^{\prime}: 2,3\right]$ pyrimido[1,6-a]quinazoline-3,5-dione (8d)

The compound was obtained from the reaction of $(5 \mathbf{b})$ and benzaldehyde $(1.06 \mathrm{~g}, 10 \mathrm{mmol})$ with chloroacetic acid or compound $(\mathbf{7 b})$ with benzaldehyde, as yellow crystals, crystallized from acetone in 79\% yield, m.p. $>350^{\circ} \mathrm{C}$. IR $\left(v, \mathrm{~cm}^{-1}\right) \mathrm{KBr}: 3060$ (CH-aryl), 2950 (CH-aliph), 1688, 1678 (2CO, amide), $1638(\mathrm{C}=\mathrm{N}) .{ }^{1} \mathrm{H}-\mathrm{NMR}\left(\mathrm{DMSO}-d_{6}, \mathrm{ppm}\right) \delta 2.33\left(\mathrm{~s}, 3 \mathrm{H}, \mathrm{CH}_{3}\right), 3.97\left(\mathrm{~s}, 6 \mathrm{H}, 2 \mathrm{OCH}_{3}\right), 5.60(\mathrm{~s}, 1 \mathrm{H}$, $\mathrm{CH}$, thiazole), $6.74(\mathrm{~d}, 1 \mathrm{H}, J=2.30 \mathrm{~Hz}$, furan), $7.34(\mathrm{~s}, 1 \mathrm{H}$, pyrimidine), $7.45-7.65(\mathrm{~m}, 5 \mathrm{H}$, phenyl), 7.74 $\left(\mathrm{d}, 1 \mathrm{H}, J=2.35 \mathrm{~Hz}\right.$, furan), $8.02(\mathrm{~s}, 1 \mathrm{H}, \mathrm{CH}) ;{ }^{13} \mathrm{C}-\mathrm{NMR}$ (DMSO-d 6$) \delta 23.5\left(1 \mathrm{C}, \mathrm{CH}_{3}\right), 61.8\left(2 \mathrm{C}, 2 \mathrm{OCH}_{3}\right)$, 77.7 (1C, $\mathrm{CH}$, thiazole), 91.7 (1C, $\mathrm{CH}$, pyrimidine), 101.4, 105.3, 108.8, 120.7 (4C, benzo furane), 122.2 (1C, CH), 126.1 (1C, pyrimidine), 127.7, 128.3, 128.8, 134.6 (6C, phenyl), 137.7 (1C, thiazole), 146.2, 146.7, 151.9, 160.6, 164.8 (5C, pyrimidobenzofurane), 165.1, 168.3 (2C, 2C=O); MS (70 eV, \%) m/z $473\left(\mathrm{M}^{+}\right.$, 84\%); Anal. Calc. (Found) for $\mathrm{C}_{25} \mathrm{H}_{19} \mathrm{~N}_{3} \mathrm{O}_{5} \mathrm{~S}$ (473.50): C, 63.42 (63.50); H, 4.04 (4.10); N, 8.87 (8.80). 
3.19. Synthesis of 2-(4-Chlorobenzylidene)-9,13-dimethoxy-8-methyl-2-hydro-5H,14aH-furo[3,2-g]thiazolo $\left[2^{\prime}, 3^{\prime}: 2,3\right]$ pyrimido[1,6-a]quinazoline-3,5-dione (8e)

The compound was obtained from the reaction of $(5 \mathbf{b})$ and 4-chlorobenzaldehyde $(1.40 \mathrm{~g}, 10 \mathrm{mmol})$ with chloroacetic acid or compound (7b) with 4-chlorobenzaldehyde, as yellowish crystals, crystallized from methanol in 88\% yield, m.p. > $350{ }^{\circ} \mathrm{C}$. IR $\left(v, \mathrm{~cm}^{-1}\right) \mathrm{KBr}: 3055$ (CH-aryl), 2945 (CH-aliph), 1686, $1674\left(2 \mathrm{CO}\right.$, amide), $1634(\mathrm{C}=\mathrm{N}) .{ }^{1} \mathrm{H}-\mathrm{NMR}\left(\mathrm{DMSO}-d_{6}, \mathrm{ppm}\right) \delta 2.30\left(\mathrm{~s}, 3 \mathrm{H}, \mathrm{CH}_{3}\right), 3.98\left(\mathrm{~s}, 6 \mathrm{H}, 2 \mathrm{OCH}_{3}\right)$, 5.59 (s, 1H, CH, thiazole), $6.73(\mathrm{~d}, 1 \mathrm{H}, \mathrm{J}=2.31 \mathrm{~Hz}$, furan), 7.35 (s, 1H, pyrimidine), 7.47-7.52 (dd, 2H, $J=7.68,7.62 \mathrm{~Hz}$, 4-chlorophenyl), 7.57-7.62 (dd, 2H, J = 7.61, 7.65 Hz, 4-chloro phenyl), $7.75(\mathrm{~d}, 1 \mathrm{H}$, $J=2.36 \mathrm{~Hz}$, furan), $8.04(\mathrm{~s}, 1 \mathrm{H}, \mathrm{CH}) ;{ }^{13} \mathrm{C}-\mathrm{NMR}\left(\mathrm{DMSO}-d_{6}\right) \delta 23.7\left(1 \mathrm{C}, \mathrm{CH}_{3}\right), 61.9\left(2 \mathrm{C}, 2 \mathrm{OCH}_{3}\right), 77.6(1 \mathrm{C}$, $\mathrm{CH}$, thiazole), 91.2 (1C, CH, pyrimidine), 100.5, 105.6, 108.4, 120.8 (4C, benzo furane), $122.4(1 \mathrm{C}, \mathrm{CH})$, 126.3 (1C, pyrimidine), 128.2, 128.8, 131.5, 131.9 (6C, 4-chloro phenyl), 137.5 (1C, thiazole), 146.3, 146.9, 150.8, 160.4, 164.9 (5C, pyrimidobenzofurane), 165.3, 168.1 (2C, 2C=O); MS (70 eV, \%) m/z $507\left(\mathrm{M}^{+}\right.$, 98\%); Anal. Calc. (Found) for $\mathrm{C}_{25} \mathrm{H}_{18} \mathrm{ClN}_{3} \mathrm{O}_{5} \mathrm{~S}$ (507.94): C, 59.12 (59.22); H, 3.57 (3.50); N, 8.27 (8.35).

3.20. Synthesis of 9,13-Dimethoxy-2-(4-methoxybenzylidene)-8-methyl-2-hydro-5H,14aH-furo[3,2-g]thiazolo $\left[2^{\prime}, 3^{\prime}: 2,3\right]$ pyrimido $[1,6-a]$ quinazoline-3,5-dione (8f)

The compound was obtained from the reaction of $(5 \mathbf{b})$ and 4-methoxy-benzaldehyde (1.36 $\mathrm{g}$, $10 \mathrm{mmol}$ ) with chloroacetic acid or compound (7b) with 4-methoxybenzaldehyde, as brownish crystals, crystallized from benzene in 73\% yield, m.p. $>350{ }^{\circ} \mathrm{C}$. IR $\left(v, \mathrm{~cm}^{-1}\right) \mathrm{KBr}: 3052(\mathrm{CH}-\operatorname{aryl}), 2944$ (CH-aliph), 1685, 1672 (2CO, amide), 1630 (C=N). ${ }^{1} \mathrm{H}-\mathrm{NMR}$ (DMSO-d, $\left.\mathrm{ppm}\right) \delta 2.28\left(\mathrm{~s}, 3 \mathrm{H}, \mathrm{CH}_{3}\right), 3.95$ $\left(\mathrm{s}, 6 \mathrm{H}, 2 \mathrm{OCH}_{3}\right), 4.12\left(\mathrm{~s}, 3 \mathrm{H}, \mathrm{OCH}_{3}\right), 5.55(\mathrm{~s}, 1 \mathrm{H}, \mathrm{CH}$, thiazole), $6.71(\mathrm{~d}, 1 \mathrm{H}, \mathrm{J}=2.32 \mathrm{~Hz}$, furan), $7.37(\mathrm{~s}, 1 \mathrm{H}$, pyrimidine), 7.49-7.54 (dd, $2 \mathrm{H}, \mathrm{J}=7.65,7.61 \mathrm{~Hz}$, 4-methoxyphenyl), 7.58-7.63 (dd, 2H, J = 7.62, 7.66 Hz, 4-methoxyphenyl), $7.76\left(\mathrm{~d}, 1 \mathrm{H}, J=2.35 \mathrm{~Hz}\right.$, furan), $8.06(\mathrm{~s}, 1 \mathrm{H}, \mathrm{CH}) ;{ }^{13} \mathrm{C}-\mathrm{NMR}\left(\mathrm{DMSO}-d_{6}\right) \delta 23.4(1 \mathrm{C}$, $\left.\mathrm{CH}_{3}\right), 58.2\left(1 \mathrm{C}, \mathrm{OCH}_{3}\right), 61.8\left(2 \mathrm{C}, 2 \mathrm{OCH}_{3}\right), 77.3(1 \mathrm{C}, \mathrm{CH}$, thiazole $), 91.5(1 \mathrm{C}, \mathrm{CH}$, pyrimidine $), 100.2$, 105.5, 108.6, 120.5 (4C, benzofurane), 122.7 (1C, CH), 126.1 (1C, pyrimidine), 127.2, 128.4, 129.1, 152.2 (6C, 4-methoxyphenyl), 137.8 (1C, thiazole), 146.1, 146.7, 150.6, 160.5, 164.8 (5C, pyrimidobenzo furane), 165.7, $168.2(2 \mathrm{C}, 2 \mathrm{C}=\mathrm{O})$; $\mathrm{MS}(70 \mathrm{eV}, \%) \mathrm{m} / \mathrm{z} 503\left(\mathrm{M}^{+}, 91 \%\right)$; Anal. Calc. (Found) for $\mathrm{C}_{26} \mathrm{H}_{21} \mathrm{~N}_{3} \mathrm{O}_{6} \mathrm{~S}$ (503.53): C, 62.02 (62.10); H, 4.20 (4.28); N, 8.35 (8.27).

\subsection{General Procedure for Synthesis of 3-(((7-Methoxy or 7,11-dimethoxy)-6-methyl-3-oxo-3H-furo[3,2-g]} pyrimido[1,6-a] quinazolin-1-yl)thio)pentane-2,4-dione (9a,b)

To a warmed ethanolic potassium hydroxide solution (prepared by dissolving $10 \mathrm{mmol}$ of $\mathrm{KOH}$ in $50 \mathrm{~mL}$ ethanol) was added each of $5 \mathrm{a}(3.13 \mathrm{~g}, 0.01 \mathrm{~mol})$ or $5 \mathbf{b}(3.43 \mathrm{~g}, 0.01 \mathrm{~mol})$, the heating was continued for $40 \mathrm{~min}$ and the mixture was allowed to cool to room temperature, and the proper 3-chloro-pentane-2,4-dione (3-chloroacetylacetone, $1.12 \mathrm{~mL}, 0.01 \mathrm{~mol}$ ) was added. The mixture was stirred under reflux for 6-8 h (control TLC), and then cool to room temperature, poured into cold water $(100 \mathrm{~mL})$. The solid product precipitated was filtered off, washed with $100 \mathrm{~mL}$ water; the product was dried and crystallized from the suitable solvent to afford $\mathbf{9 a}$ and $\mathbf{9 b}$ in good yields, respectively.

3.22. Synthesis of 3-((7-Methoxy-6-methyl-3-oxo-3H-furo[3,2-g]pyrimido[1,6-a]quinazolin-1-yl)thio)pentane2,4-dione (9a)

The compound was obtained from the reaction of (5a) with 3-chloro-pentane-2,4-dione (3-chloroacetylacetone), as white crystals, crystallized from hexane in 92\% yield, m.p. $>350{ }^{\circ} \mathrm{C}$. IR ( $\left.v, \mathrm{~cm}^{-1}\right) \mathrm{KBr}: 3035$ (CH-aryl), 2945 (CH-aliph), 1725, 1721 (2CO, acetyl), 1682 (CO, amide), 1638 $(\mathrm{C}=\mathrm{N}) .{ }^{1} \mathrm{H}-\mathrm{NMR}\left(\mathrm{DMSO}-d_{6}, \mathrm{ppm}\right) \delta 2.26\left(\mathrm{~s}, 6 \mathrm{H}, 2 \mathrm{COCH}_{3}\right), 2.34\left(\mathrm{~s}, 3 \mathrm{H}, \mathrm{CH}_{3}\right), 3.90\left(\mathrm{~s}, 3 \mathrm{H}, \mathrm{OCH}_{3}\right), 4.08$ (s, 1H, CH), $6.73(\mathrm{~d}, 1 \mathrm{H}, J=2.31 \mathrm{~Hz}$, furan), $7.37(\mathrm{~s}, 1 \mathrm{H}$, pyrimidine), $7.62(\mathrm{~s}, 1 \mathrm{H}$, phenyl), $7.75(\mathrm{~d}, 1 \mathrm{H}$, $J=2.33 \mathrm{~Hz}$, furan), ${ }^{13} \mathrm{C}-\mathrm{NMR}\left(\mathrm{DMSO}-d_{6}\right) \delta 23.8\left(1 \mathrm{C}, \mathrm{CH}_{3}\right), 26.5\left(2 \mathrm{C}, 2 \mathrm{COCH}_{3}\right), 61.6\left(1 \mathrm{C}, \mathrm{OCH}_{3}\right), 68.2$ (1C, CH), 91.3 (1C, CH, phenyl), 96.5 (1C, CH, pyrimidine), 100.1, 105.3, 108.5, 142.6, 146.1, 153.5, 157.4, 160.3, 163.8, 165.7 (10C, Ar-C), 168.6, 184.5, 188.2 (3C, 3C=O); MS (70 eV, \%) m/z $411\left(\mathrm{M}^{+}, 95 \%\right)$; Anal. Calc. (Found) for $\mathrm{C}_{20} \mathrm{H}_{17} \mathrm{~N}_{3} \mathrm{O}_{5} \mathrm{~S}$ (411.43): C, 58.39 (58.32); H, 4.16 (4.10); N, 10.21 (10.16). 
3.23. Synthesis of 3-((7,11-Dimethoxy-6-methyl-3-oxo-3H-furo[3,2-g]pyrimido[1,6-a]quinazolin-1-yl)thio) pentane-2,4-dione (9b)

The compound was obtained from the reaction of (5b) with 3-chloro-pentane-2,4-dione (3-chloroacetylacetone), as white crystals, crystallized from dioxane in $90 \%$ yield, m.p. $348^{\circ} \mathrm{C}$. IR ( $v$, $\mathrm{cm}^{-1}$ ) KBr: 3038 (CH-aryl), 2949 (CH-aliph), 1728, 1722 (2CO, acetyl), 1684 (CO, amide), 1636 (C=N). ${ }^{1} \mathrm{H}-\mathrm{NMR}\left(\mathrm{DMSO}-d_{6}, \mathrm{ppm}\right) \delta 2.28\left(\mathrm{~s}, 6 \mathrm{H}, 2 \mathrm{COCH}_{3}\right), 2.37\left(\mathrm{~s}, 3 \mathrm{H}, \mathrm{CH}_{3}\right), 3.98\left(\mathrm{~s}, 6 \mathrm{H}, 2 \mathrm{OCH}_{3}\right), 4.15(\mathrm{~s}$, $1 \mathrm{H}, \mathrm{CH}), 6.72(\mathrm{~d}, 1 \mathrm{H}, J=2.34 \mathrm{~Hz}$, furan), 7.35 (s, 1H, pyrimidine), $7.79(\mathrm{~d}, 1 \mathrm{H}, J=2.37 \mathrm{~Hz}$, furan), ${ }^{13} \mathrm{C}-\mathrm{NMR}\left(\mathrm{DMSO}-d_{6}\right) \delta 23.4\left(1 \mathrm{C}, \mathrm{CH}_{3}\right), 26.1\left(2 \mathrm{C}, 2 \mathrm{COCH}_{3}\right), 61.9\left(2 \mathrm{C}, 2 \mathrm{OCH}_{3}\right), 68.5(1 \mathrm{C}, \mathrm{CH}), 97.1(1 \mathrm{C}$, $\mathrm{CH}$, pyrimidine), 100.2, 105.7, 108.9, 122.5, 130.2, 146.3, 146.8, 150.7, 159.6, 164.2, 166.4 (11C, Ar-C), 168.8, 185.1, $188.7(3 \mathrm{C}, 3 \mathrm{C}=\mathrm{O})$; $\mathrm{MS}(70 \mathrm{eV}, \%) \mathrm{m} / \mathrm{z} 441\left(\mathrm{M}^{+}, 90 \%\right)$; Anal. Calc. (Found) for $\mathrm{C}_{21} \mathrm{H}_{19} \mathrm{~N}_{3} \mathrm{O}_{6} \mathrm{~S}$ (441.46): C, 57.14 (57.20); H, 4.34 (4.39); N, 9.52 (9.60).

3.24. General Procedure for Synthesis of 1-((3,5-Dimethyl-1H-pyrazol-4-yl)thio)-(7-methoxy or 7,11dimethoxy)-6-methyl-3H-furo [3,2-g]pyrimido[1,6-a]quinazolin-3-one (10a,b)

A mixture of $9 \mathrm{a}(4.11 \mathrm{~g}, 0.01 \mathrm{~mol})$ or $9 \mathrm{~b}(4.41 \mathrm{~g}, 0.01 \mathrm{~mol})$, and hydrazine hydrate $(99-100 \%)$ in dioxane $(30 \mathrm{~mL})$ and ethanol $(20 \mathrm{~mL})$ was stirred under reflux for $13-15 \mathrm{~h}$. The reaction mixture was allowed to cool to room temperature, poured into cold water $(100 \mathrm{~mL})$. The deposited precipitate was filtered off, dried, and crystallized from the proper solvent to give 10a and 10b in good yields, respectively.

3.25. Synthesis of 1-((3,5-Dimethyl-1H-pyrazol-4-yl)thio)-7-methoxy-6-methyl-3H-furo[3,2-g]pyrimido [1,6-a] quinazolin-3-one (10a)

The compound was obtained from the reaction of (9a) with hydrazine hydrate, as yellow crystals, crystallized from dimethylformamide in $95 \%$ yield, m.p. $>350{ }^{\circ} \mathrm{C}$. IR $\left(v, \mathrm{~cm}^{-1}\right) \mathrm{KBr}: 3375$ (brs, NH), 3030 (CH-aryl), 2940 (CH-aliph), 1684 (CO, amide), 1633 (C=N). ${ }^{1} \mathrm{H}-\mathrm{NMR}$ (DMSO-d $\left.d_{6}, \mathrm{ppm}\right) \delta 2.28$ (s, $\left.3 \mathrm{H}, \mathrm{CH}_{3}\right), 2.38\left(\mathrm{~s}, 3 \mathrm{H}, \mathrm{CH}_{3}\right), 2.40\left(\mathrm{~s}, 3 \mathrm{H}, \mathrm{CH}_{3}\right), 3.92\left(\mathrm{~s}, 3 \mathrm{H}, \mathrm{OCH}_{3}\right), 6.75(\mathrm{~d}, 1 \mathrm{H}, J=2.32 \mathrm{~Hz}$, furan $)$, 7.32 (s, 1H, pyrimidine), $7.60\left(\mathrm{~s}, 1 \mathrm{H}\right.$, phenyl), $7.72\left(\mathrm{~d}, 1 \mathrm{H}, \mathrm{J}=2.37 \mathrm{~Hz}\right.$, furan), 11.50 (brs, NH, $\mathrm{D}_{2} \mathrm{O}$ exchangeable); ${ }^{13} \mathrm{C}-\mathrm{NMR}\left(\mathrm{DMSO}-d_{6}\right) \delta 20.1,20.3,23.2\left(3 \mathrm{C}, 3 \mathrm{CH}_{3}\right), 61.4\left(1 \mathrm{C}, \mathrm{OCH}_{3}\right), 91.1(1 \mathrm{C}, \mathrm{CH}$, phenyl), 96.3 (1C, CH, pyrimidine), 100.2, 105.1, 107.2, 108.4, 142.3, 144.5, 146.6, 154.1, 158.2, 160.5, 164.4, 166.3 (13C, Ar-C), 168.8 (1C, C=O); MS (70 eV, \%) m/z 407 ( $\left.\mathrm{M}^{+}, 100 \%\right)$; Anal. Calc. (Found) for $\mathrm{C}_{20} \mathrm{H}_{17} \mathrm{~N}_{5} \mathrm{O}_{3} \mathrm{~S}$ (407.45): C, 58.96 (58.88); H, 4.21 (4.15); N, 17.19 (17.10).

3.26. Synthesis of 1-((3,5-Dimethyl-1H-pyrazol-4-yl)thio)-7,11-dimethoxy-6-methyl-3H-furo[3,2-g]pyrimido [1,6-a]quinazolin-3-one (10b)

The compound was obtained from the reaction of $(\mathbf{9 b})$ with hydrazine hydrate, as yellowish crystals, crystallized from dioxane in $91 \%$ yield, m.p. $>350{ }^{\circ} \mathrm{C}$. IR ( $\left.v, \mathrm{~cm}^{-1}\right) \mathrm{KBr}: 3370$ (brs, NH), 3032 (CH-aryl), 2943 (CH-aliph), 1682 (CO, amide), 1635 (C=N). ${ }^{1} \mathrm{H}-\mathrm{NMR}\left(\mathrm{DMSO}-d_{6}, \mathrm{ppm}\right) \delta 2.30(\mathrm{~s}, 3 \mathrm{H}$, $\left.\mathrm{CH}_{3}\right), 2.39\left(\mathrm{~s}, 3 \mathrm{H}, \mathrm{CH}_{3}\right), 2.41\left(\mathrm{~s}, 3 \mathrm{H}, \mathrm{CH}_{3}\right), 3.98\left(\mathrm{~s}, 6 \mathrm{H}, 2 \mathrm{OCH}_{3}\right), 6.77(\mathrm{~d}, 1 \mathrm{H}, \mathrm{J}=2.36 \mathrm{~Hz}$, furan $), 7.38$ (s, 1H, pyrimidine), 7.79 (d, 1H, $J=2.34 \mathrm{~Hz}$, furan), 11.55 (brs, NH, $\mathrm{D}_{2} \mathrm{O}$ exchangeable); ${ }^{13} \mathrm{C}-\mathrm{NMR}$ $\left(\mathrm{DMSO}_{6}\right) \delta 20.4,20.6,23.5\left(3 \mathrm{C}, 3 \mathrm{CH}_{3}\right), 61.9\left(2 \mathrm{C}, 2 \mathrm{OCH}_{3}\right), 96.7$ (1C, CH, pyrimidine), 100.3, 105.6, $107.8,108.5,122.5,130.4,144.8,146.1,146.8,151.4,160.5,164.2,166.6$ (14C, Ar-C), 168.9 (1C, C=O); MS $(70 \mathrm{eV}, \%) \mathrm{m} / z 437\left(\mathrm{M}^{+}, 100 \%\right) ;$ Anal. Calc. (Found) for $\mathrm{C}_{21} \mathrm{H}_{17} \mathrm{~N}_{5} \mathrm{O}_{4} \mathrm{~S}$ (437.47): C, 57.66 (57.72); $\mathrm{H}, 4.38$ (4.30); N, 16.01 (16.10).

3.27. General Procedure for Synthesis of 1-((4,6-Dimethyl-2-(oxo or thioxo)-1,2-dihydropyrimidin-5-yl)thio)(7-methoxy or 7,11-dimethoxy)-6-methyl-3H-furo[3,2-g]pyrimido[1,6-a]quinazolin-3-one (11a-d)

A mixture of $9 \mathrm{a}(4.11 \mathrm{~g}, 0.01 \mathrm{~mol})$ or $9 \mathrm{~b}(4.41 \mathrm{~g}, 0.01 \mathrm{~mol})$, and urea $(0.60 \mathrm{~g}, 0.01 \mathrm{~mol})$ or thiourea $(0.76 \mathrm{~g}, 0.01 \mathrm{~mol})$ was stirred under reflux in dioxane $(40 \mathrm{~mL})$ in the presence of catalytic amount of piperidine $(1 \mathrm{~mL})$ for $16-18 \mathrm{~h}$. The reaction mixture was allowed to cool to room temperature, poured 
into water $(100 \mathrm{~mL})$, the deposited precipitate was filtered off, washed with ethanol $(40 \mathrm{~mL})$, dried and crystallized from proper solvent to afford (11a-d).

3.28. Synthesis of 1-((4,6-Dimethyl-2-oxo-1,2-dihydropyrimidin-5-yl)thio)-7-methoxy-6-methyl-3Hfuro[3,2-g]pyrimido[1,6-a]quinazolin-3-one (11a)

The compound was obtained from the reaction of $(\mathbf{9 a})$ with urea, as yellowish crystals, crystallized from dioxane in $86 \%$ yield, m.p. $>350{ }^{\circ} \mathrm{C}$. IR $\left(v, \mathrm{~cm}^{-1}\right) \mathrm{KBr}: 3380$ (brs, NH), $3032(\mathrm{CH}$-aryl), 2942 (CH-aliph), 1685, 1680 (2C=O, amide), $1635(\mathrm{C}=\mathrm{N}) .{ }^{1} \mathrm{H}-\mathrm{NMR}$ (DMSO- $\left.d_{6}, \mathrm{ppm}\right) \delta 2.11\left(\mathrm{~s}, 3 \mathrm{H}, \mathrm{CH}_{3}\right)$, $2.28\left(\mathrm{~s}, 3 \mathrm{H}, \mathrm{CH}_{3}\right), 2.37\left(\mathrm{~s}, 3 \mathrm{H}, \mathrm{CH}_{3}\right), 3.94\left(\mathrm{~s}, 3 \mathrm{H}, \mathrm{OCH}_{3}\right), 6.72(\mathrm{~d}, 1 \mathrm{H}, J=2.31 \mathrm{~Hz}$, furan), $7.36(\mathrm{~s}, 1 \mathrm{H}$, pyrimidine), $7.63\left(\mathrm{~s}, 1 \mathrm{H}\right.$, phenyl), $7.79\left(\mathrm{~d}, 1 \mathrm{H}, J=2.38 \mathrm{~Hz}\right.$, furan), 10.60 (brs, $\mathrm{NH}, \mathrm{D}_{2} \mathrm{O}$ exchangeable); ${ }^{13} \mathrm{C}-\mathrm{NMR}\left(\mathrm{DMSO}-d_{6}\right) \delta 20.6,20.9,23.5\left(3 \mathrm{C}, 3 \mathrm{CH}_{3}\right), 61.8\left(1 \mathrm{C}, \mathrm{OCH}_{3}\right), 85.4(1 \mathrm{C}$, pyrimidine), 91.5 (1C, $\mathrm{CH}$, phenyl), 97.7 (1C, CH, pyrimidine), 100.4, 105.7, 108.2, 143.1, 146.1, 153.6, 158.5, 160.4, 163.8, 164.7, 165.2, 166.8 (12C, Ar-C), 169.2, 172.5 (2C, 2C=O); MS (70 eV, \%) m/z $435\left(\mathrm{M}^{+}, 92 \%\right)$; Anal. Calc. (Found) for $\mathrm{C}_{21} \mathrm{H}_{17} \mathrm{~N}_{5} \mathrm{O}_{4} \mathrm{~S}$ (435.46): $\mathrm{C}, 57.92$ (57.85); $\mathrm{H}, 3.94$ (3.99); N, 16.08 (16.15).

3.29. Synthesis of 1-((4,6-Dimethyl-2-thioxo-1,2-dihydropyrimidin-5-yl)thio)-7-methoxy-6-methyl-3Hfuro[3,2-g]pyrimido[1,6-a]quinazolin-3-one (11b)

The compound was obtained from the reaction of (9a) with thiourea, as yellow crystals, crystallized from dimethylformamide in $82 \%$ yield, m.p. $>350{ }^{\circ} \mathrm{C}$. IR $\left(v, \mathrm{~cm}^{-1}\right) \mathrm{KBr}$ : 3385 (brs, $\mathrm{NH}), 3035$ (CH-aryl), 2944 (CH-aliph), 1681 (C=O, amide), $1632(\mathrm{C}=\mathrm{N}) .{ }^{1} \mathrm{H}-\mathrm{NMR}$ (DMSO-d $\left.d_{6}, \mathrm{ppm}\right)$ $\delta 2.10\left(\mathrm{~s}, 3 \mathrm{H}, \mathrm{CH}_{3}\right), 2.27\left(\mathrm{~s}, 3 \mathrm{H}, \mathrm{CH}_{3}\right), 2.36\left(\mathrm{~s}, 3 \mathrm{H}, \mathrm{CH}_{3}\right), 3.91\left(\mathrm{~s}, 3 \mathrm{H}, \mathrm{OCH}_{3}\right), 6.70(\mathrm{~d}, 1 \mathrm{H}, J=2.30 \mathrm{~Hz}$, furan), $7.34(\mathrm{~s}, 1 \mathrm{H}$, pyrimidine), $7.61(\mathrm{~s}, 1 \mathrm{H}$, phenyl), $7.78(\mathrm{~d}, 1 \mathrm{H}, J=2.36 \mathrm{~Hz}$, furan), 12.30 (brs, NH, $\mathrm{D}_{2} \mathrm{O}$ exchangeable); ${ }^{13} \mathrm{C}-\mathrm{NMR}\left(\mathrm{DMSO}-d_{6}\right) \delta 21.4,21.8,23.7\left(3 \mathrm{C}, 3 \mathrm{CH}_{3}\right), 61.7\left(1 \mathrm{C}, \mathrm{OCH}_{3}\right), 84.6(1 \mathrm{C}$, pyrimidine), 91.8 (1C, $\mathrm{CH}$, phenyl), 98.2 (1C, $\mathrm{CH}$, pyrimidine), 100.2, 105.8, 108.1, 143.7, 146.3, 153.8, 158.9, 160.5, 163.9, 164.2, 165.8, 169.6 (12C, Ar-C), 170.1 (1C, C=O), 177.8 (1C, C=S); MS (70 eV, \%) m/z $451\left(\mathrm{M}^{+}, 90 \%\right.$ ); Anal. Calc. (Found) for $\mathrm{C}_{21} \mathrm{H}_{17} \mathrm{~N}_{5} \mathrm{O}_{3} \mathrm{~S}_{2}$ (451.52): $\mathrm{C}, 55.86$ (55.78); $\mathrm{H}, 3.80$ (3.88); N, $15.51(15.60)$.

3.30. Synthesis of 1-((4,6-Dimethyl-2-oxo-1,2-dihydropyrimidin-5-yl)thio)-7,11-dimethoxy-6-methyl-3Hfuro[3,2-g]pyrimido[1,6-a]quinazolin-3-one (11c)

The compound was obtained from the reaction of $(\mathbf{9 b})$ with urea, as yellow crystals, crystallized from methanol in $80 \%$ yield, m.p. $>350^{\circ} \mathrm{C}$. IR $\left(v, \mathrm{~cm}^{-1}\right) \mathrm{KBr}: 3377$ (brs, NH), 3039 (CH-aryl), 2937 (CH-aliph), 1687, 1683 (2C=O, amide), $1638(\mathrm{C}=\mathrm{N}) .{ }^{1} \mathrm{H}-\mathrm{NMR}$ (DMSO- $\left.d_{6}, \mathrm{ppm}\right) \delta 2.09\left(\mathrm{~s}, 3 \mathrm{H}, \mathrm{CH}_{3}\right)$, $2.27\left(\mathrm{~s}, 3 \mathrm{H}, \mathrm{CH}_{3}\right), 2.33\left(\mathrm{~s}, 3 \mathrm{H}, \mathrm{CH}_{3}\right), 3.99\left(\mathrm{~s}, 6 \mathrm{H}, 2 \mathrm{OCH}_{3}\right), 6.75(\mathrm{~d}, 1 \mathrm{H}, J=2.32 \mathrm{~Hz}$, furan), $7.38(\mathrm{~s}, 1 \mathrm{H}$, pyrimidine), $7.76\left(\mathrm{~d}, 1 \mathrm{H}, J=2.37 \mathrm{~Hz}\right.$, furan), 10.74 (brs, $\mathrm{NH}, \mathrm{D}_{2} \mathrm{O}$ exchangeable); ${ }^{13} \mathrm{C}-\mathrm{NMR}$ (DMSO- $d_{6}$ ) $\delta$ 20.5, 20.7, $23.7\left(3 \mathrm{C}, 3 \mathrm{CH}_{3}\right), 61.9\left(2 \mathrm{C}, 2 \mathrm{OCH}_{3}\right), 87.1$ (1C, pyrimidine), 98.9 (1C, $\mathrm{CH}$, pyrimidine), 100.2, 105.4, 108.8, 122.3, 130.5, 146.1, 146.9, 152.1, 158.2, 164.1, 164.5, 166.2, 168.5 (13C, Ar-C), 169.8, 171.4 $(2 \mathrm{C}, 2 \mathrm{C}=\mathrm{O})$; $\mathrm{MS}(70 \mathrm{eV}, \%) \mathrm{m} / \mathrm{z} 465\left(\mathrm{M}^{+}, 88 \%\right.$ ); Anal. Calc. (Found) for $\mathrm{C}_{22} \mathrm{H}_{19} \mathrm{~N}_{5} \mathrm{O}_{5} \mathrm{~S}$ (465.48): $\mathrm{C}, 56.77$ (56.70); H, 4.11 (4.05); N, 15.05 (15.14).

3.31. Synthesis of 1-((4, 6-Dimethyl-2-thioxo-1,2-dihydropyrimidin-5-yl)thio)-7,11-dimethoxy-6-methyl-3Hfuro[3,2-g]pyrimido[1,6-a]quinazolin-3-one (11d)

The compound was obtained from the reaction of $(\mathbf{9 b})$ with thiourea, as yellowish crystals, crystallized from dioxane in $80 \%$ yield, m.p. $>350^{\circ} \mathrm{C}$. IR $\left(v, \mathrm{~cm}^{-1}\right) \mathrm{KBr}$ : 3388 (brs, NH), 3034 (CH-aryl), 2945 (CH-aliph), $1683\left(\mathrm{C}=\mathrm{O}\right.$, amide), $1630(\mathrm{C}=\mathrm{N}) .{ }^{1} \mathrm{H}-\mathrm{NMR}$ (DMSO-d $\left.d_{6}, \mathrm{ppm}\right) \delta 2.13\left(\mathrm{~s}, 3 \mathrm{H}, \mathrm{CH}_{3}\right)$, $2.25\left(\mathrm{~s}, 3 \mathrm{H}, \mathrm{CH}_{3}\right), 2.32\left(\mathrm{~s}, 3 \mathrm{H}, \mathrm{CH}_{3}\right), 4.01\left(\mathrm{~s}, 6 \mathrm{H}, 2 \mathrm{OCH}_{3}\right), 6.73(\mathrm{~d}, 1 \mathrm{H}, J=2.34 \mathrm{~Hz}$, furan), $7.36(\mathrm{~s}, 1 \mathrm{H}$, pyrimidine), $7.80\left(\mathrm{~d}, 1 \mathrm{H}, J=2.38 \mathrm{~Hz}\right.$, furan), 12.50 (brs, $\mathrm{NH}, \mathrm{D}_{2} \mathrm{O}$ exchangeable); ${ }^{13} \mathrm{C}-\mathrm{NMR}$ (DMSO- $d_{6}$ ) $\delta$ 21.5, 21.9, $23.8\left(3 \mathrm{C}, 3 \mathrm{CH}_{3}\right), 62.01\left(2 \mathrm{C}, 2 \mathrm{OCH}_{3}\right), 86.1$ (1C, pyrimidine), 99.3 (1C, $\mathrm{CH}$, pyrimidine), 100.4, 105.2, 108.7, 122.7, 130.4, 146.3, 146.7, 151.9, 159.1, 164.3, 164.5, 166.9, 168.2 (13C, Ar-C), 169.8(1C, C=O), 
178.5 (1C, C=S); $\mathrm{MS}(70 \mathrm{eV}, \%) \mathrm{m} / \mathrm{z} 481\left(\mathrm{M}^{+}, 86 \%\right.$ ); Anal. Calc. (Found) for $\mathrm{C}_{22} \mathrm{H}_{19} \mathrm{~N}_{5} \mathrm{O}_{4} \mathrm{~S}_{2}$ (481.54): $\mathrm{C}$, 54.87 (54.95); H, 3.98 (3.90); N, 14.54 (14.47).

3.32. General Procedure for Synthesis of (7-Methoxy or 7,11-dimethoxy)-6-methyl-1-(methylthio)-3Hfuro[3,2-g]pyrimido[1,6-a]quinazolin-3-one (12a,b)

To a warmed ethanolic $\mathrm{KOH}$ solution (prepared by dissolving $10 \mathrm{mmol}$ of $\mathrm{KOH}$ in $50 \mathrm{~mL}$ ethanol) was added each of $5 \mathbf{a}(3.13 \mathrm{~g}, 0.01 \mathrm{~mol})$ or $\mathbf{5 b}(3.43 \mathrm{~g}, 0.01 \mathrm{~mol})$, the heating was continued for $30 \mathrm{~min}$ and the mixture was allowed to cool to room temperature, and methyl iodide $(0.62 \mathrm{~mL}, 0.01 \mathrm{~mol})$ was added. The mixture was stirred under reflux for $5-7 \mathrm{~h}$, then cool to room temperature, poured into cold water $(100 \mathrm{~mL})$. The solid product precipitated was filtered off, washed with water; the product was dried and crystallized from the proper solvent to produce 12a and 12b, respectively.

\subsection{Synthesis of 7-Methoxy-6-methyl-1-(methylthio)-3H-furo[3,2-g]pyrimido[1,6-a]quinazolin-3-one (12a)}

The compound was obtained from the reaction of (5a) with methyl iodide, as yellow crystals, crystallized from methanol in $90 \%$ yield, m.p. $329^{\circ} \mathrm{C}$. IR $\left(v, \mathrm{~cm}^{-1}\right) \mathrm{KBr}$ : 3035 (CH-aryl), 2962 (CH-aliph), 1685 (CO, amide), $1630(\mathrm{C}=\mathrm{N}) .{ }^{1} \mathrm{H}-\mathrm{NMR}$ (DMSO- $\left.d_{6}, \mathrm{ppm}\right) \delta 2.30\left(\mathrm{~s}, 3 \mathrm{H}, \mathrm{CH}_{3}\right), 2.80\left(\mathrm{~s}, 3 \mathrm{H}, \mathrm{SCH}_{3}\right), 3.90$ $\left(\mathrm{s}, 3 \mathrm{H}, \mathrm{OCH}_{3}\right), 6.73(\mathrm{~d}, 1 \mathrm{H}, \mathrm{J}=2.31 \mathrm{~Hz}$, furan $), 7.34(\mathrm{~s}, 1 \mathrm{H}$, pyrimidine $), 7.64(\mathrm{~s}, 1 \mathrm{H}$, phenyl), $7.78(\mathrm{~d}, 1 \mathrm{H}$, $J=2.35 \mathrm{~Hz}$, furan); ${ }^{13} \mathrm{C}-\mathrm{NMR}$ (DMSO- $\left.d_{6}\right) \delta 18.9\left(1 \mathrm{C}, \mathrm{SCH}_{3}\right), 23.8\left(1 \mathrm{C}, \mathrm{CH}_{3}\right), 61.4\left(1 \mathrm{C}, \mathrm{OCH}_{3}\right), 92.7(1 \mathrm{C}$, $\mathrm{CH}$, phenyl), 98.9 (1C, $\mathrm{CH}$, pyrimidine), 100.3, 105.2, 108.5, 145.2, 146.4, 153.7, 158.3, 160.9, 164.6, 167.8 (10C, Ar-C), $169.1(1 \mathrm{C}, \mathrm{C}=\mathrm{O})$; $\mathrm{MS}(70 \mathrm{eV}, \%) \mathrm{m} / \mathrm{z} 327\left(\mathrm{M}^{+}, 100 \%\right)$; Anal. Calc. (Found) for $\mathrm{C}_{16} \mathrm{H}_{13} \mathrm{~N}_{3} \mathrm{O}_{3} \mathrm{~S}$ (327.36): C, 58.71 (58.78); H, 4.00 (4.10); N, 12.84 (12.90).

\subsection{Synthesis of 7,11-Dimethoxy-6-methyl-1-(methylthio)-3H-furo[3,2-g]pyrimido[1,6-a]quinazolin- 3-one (12b)}

The compound was obtained from the reaction of $(\mathbf{5 b})$ with methyl iodide, as yellowish crystals, crystallized from ethanol in $86 \%$ yield, m.p. $311^{\circ} \mathrm{C}$. IR $\left(v, \mathrm{~cm}^{-1}\right) \mathrm{KBr}: 3038$ (CH-aryl), 2965 (CH-aliph), $1683\left(\mathrm{CO}\right.$, amide), $1633(\mathrm{C}=\mathrm{N}) .{ }^{1} \mathrm{H}-\mathrm{NMR}$ (DMSO- $\left.d_{6}, \mathrm{ppm}\right) \delta 2.35\left(\mathrm{~s}, 3 \mathrm{H}, \mathrm{CH}_{3}\right), 2.88\left(\mathrm{~s}, 3 \mathrm{H}, \mathrm{SCH}_{3}\right), 3.97$ $\left(\mathrm{s}, 6 \mathrm{H}, 2 \mathrm{OCH}_{3}\right), 6.78(\mathrm{~d}, 1 \mathrm{H}, J=2.32 \mathrm{~Hz}$, furan), $7.38(\mathrm{~s}, 1 \mathrm{H}$, pyrimidine), $7.80(\mathrm{~d}, 1 \mathrm{H}, J=2.34 \mathrm{~Hz}$, furan); ${ }^{13} \mathrm{C}-\mathrm{NMR}$ (DMSO- $\left.d_{6}\right) \delta 19.2\left(1 \mathrm{C}, \mathrm{SCH}_{3}\right), 23.6\left(1 \mathrm{C}, \mathrm{CH}_{3}\right), 61.8\left(2 \mathrm{C}, 2 \mathrm{OCH}_{3}\right), 99.4(1 \mathrm{C}, \mathrm{CH}$, pyrimidine), 100.1, 105.7, 108.2, 122.7, 130.5, 146.1, 146.8, 151.5, 159.7, 164.4, 168.2 (11C, Ar-C), 169.7 (1C, C=O); MS $(70 \mathrm{eV}, \%) \mathrm{m} / z 357\left(\mathrm{M}^{+}, 100 \%\right)$; Anal. Calc. (Found) for $\mathrm{C}_{17} \mathrm{H}_{15} \mathrm{~N}_{3} \mathrm{O}_{4} \mathrm{~S}$ (357.38): $\mathrm{C}, 57.13$ (57.22); $\mathrm{H}, 4.23$ (4.28); N, 11.76 (11.66).

3.35. General Procedure for Synthesis of (7-Methoxy or 7,11-dimethoxy)-6-methyl-1-(methylsulfonyl)-3Hfuro[3,2-g]pyrimido[1,6-a] quinazolin-3-one (13a,b)

A mixture of $\mathbf{1 2 a}(3.27 \mathrm{~g}, 0.01 \mathrm{~mol})$ or $\mathbf{1 2} \mathbf{b}(3.57 \mathrm{~g}, 0.01 \mathrm{~mol})$, and excess amount of hydrogen peroxide $(5 \mathrm{~mL})$ in acetic acid $(40 \mathrm{~mL})$ was heated gently with stirring for 12-14 h with (TLC). The reaction mixture was allowed to cool to $0{ }^{\circ} \mathrm{C}$. The deposited precipitate was filtered off, and crystallized from the proper solvent to afford $13 \mathbf{a}$ and $\mathbf{1 3 b}$, respectively.

\subsection{Synthesis of 7-Methoxy-6-methyl-1-(methylsulfonyl)-3H-furo [3,2-g]pyrimido[1,6-a]quinazolin-} 3-one (13a)

The compound was obtained from the reaction of (12a) with hydrogen peroxide, as yellowish crystals, crystallized from benzene in $75 \%$ yield, m.p. $241^{\circ} \mathrm{C}$. IR $\left(v, \mathrm{~cm}^{-1}\right) \mathrm{KBr}: 3032(\mathrm{CH}$-aryl), 2920 (CH-aliph), 1687 (CO, amide), $1632(\mathrm{C}=\mathrm{N}), 1162,1340\left(\mathrm{SO}_{2}\right) .{ }^{1} \mathrm{H}-\mathrm{NMR}$ (DMSO- $\left.d_{6}, \mathrm{ppm}\right) \delta 2.34(\mathrm{~s}, 3 \mathrm{H}$, $\left.\mathrm{CH}_{3}\right), 2.95\left(\mathrm{~s}, 3 \mathrm{H}, \mathrm{SO}_{2} \mathrm{CH}_{3}\right), 3.92\left(\mathrm{~s}, 3 \mathrm{H}, \mathrm{OCH}_{3}\right), 6.76(\mathrm{~d}, 1 \mathrm{H}, \mathrm{J}=2.36 \mathrm{~Hz}$, furan), $7.37(\mathrm{~s}, 1 \mathrm{H}$, pyrimidine), $7.61\left(\mathrm{~s}, 1 \mathrm{H}\right.$, phenyl), $7.81\left(\mathrm{~d}, 1 \mathrm{H}, J=2.32 \mathrm{~Hz}\right.$, furan); ${ }^{13} \mathrm{C}-\mathrm{NMR}$ (DMSO- $\left.d_{6}\right) \delta 23.5\left(1 \mathrm{C}, \mathrm{CH}_{3}\right), 35.6(1 \mathrm{C}$, $\left.\mathrm{SO}_{2} \mathrm{CH}_{3}\right), 61.3\left(1 \mathrm{C}, \mathrm{OCH}_{3}\right), 93.5(1 \mathrm{C}, \mathrm{CH}$, phenyl), $99.4(1 \mathrm{C}, \mathrm{CH}$, pyrimidine), 100.2, 105.4, 108.7, 140.3, 145.5, 146.2, 153.9, 160.8, 164.4, 168.1 (10C, Ar-C), 169.5 (1C, C=O); MS (70 eV, \%) m/z $359\left(\mathrm{M}^{+}, 84 \%\right)$; Anal. Calc. (Found) for $\mathrm{C}_{16} \mathrm{H}_{13} \mathrm{~N}_{3} \mathrm{O}_{5} \mathrm{~S}$ (359.36): C, 53.48 (53.40); $\mathrm{H}, 3.65$ (3.57); N, 11.69 (11.61). 
3.37. Synthesis of 7,11-Dimethoxy-6-methyl-1-(methylsulfonyl)-3H-furo[3,2-g]pyrimido[1,6-a]quinazolin3-one (13b)

The compound was obtained from the reaction of (12b) with hydrogen peroxide, as yellow crystals, crystallized from toluene in $75 \%$ yield, m.p. $221^{\circ} \mathrm{C}$. IR ( $\left.v, \mathrm{~cm}^{-1}\right) \mathrm{KBr}: 3029$ (CH-aryl), 2918 (CH-aliph), 1684 (CO, amide), $1628(\mathrm{C}=\mathrm{N}), 1160,1342\left(\mathrm{SO}_{2}\right) .{ }^{1} \mathrm{H}-\mathrm{NMR}$ (DMSO-d, $\left.\mathrm{ppm}\right) \delta 2.29(\mathrm{~s}$, $\left.3 \mathrm{H}, \mathrm{CH}_{3}\right), 2.97\left(\mathrm{~s}, 3 \mathrm{H}, \mathrm{SO}_{2} \mathrm{CH}_{3}\right), 4.01\left(\mathrm{~s}, 6 \mathrm{H}, 2 \mathrm{OCH}_{3}\right), 6.80(\mathrm{~d}, 1 \mathrm{H}, J=2.30 \mathrm{~Hz}$, furan), $7.31(\mathrm{~s}, 1 \mathrm{H}$, pyrimidine), $7.75\left(\mathrm{~d}, 1 \mathrm{H}, J=2.33 \mathrm{~Hz}\right.$, furan); ${ }^{13} \mathrm{C}-\mathrm{NMR}\left(\mathrm{DMSO}-d_{6}\right) \delta 23.3\left(1 \mathrm{C}, \mathrm{CH}_{3}\right), 35.8\left(1 \mathrm{C}, \mathrm{SO}_{2} \mathrm{CH}_{3}\right)$, $61.9\left(2 \mathrm{C}, 2 \mathrm{OCH}_{3}\right), 99.8$ (1C, CH, pyrimidine), 100.4, 105.7, 108.9, 122.4, 130.1, 140.6, 146.3, 146.8, 151.5, 164.7, 168.5(11C, Ar-C), 169.5 (1C, C=O); MS (70 eV, \%) m/z $389\left(\mathrm{M}^{+}, 80 \%\right)$; Anal. Calc. (Found) for $\mathrm{C}_{17} \mathrm{H}_{15} \mathrm{~N}_{3} \mathrm{O}_{6} \mathrm{~S}$ (389.38): C, 52.44 (52.50); H, 3.88 (3.80); N, 10.79 (10.71).

3.38. General Procedure for Synthesis of (7-Methoxy or 7,11-dimethoxy)-6-methyl-1-((piperazin-1-yl) or morpholino)-3H-furo[3,2-g]pyrimido[1,6-a]quinazolin-3-one (14a-d)

In a warm solution of $12 \mathrm{a}(3.27 \mathrm{~g}, 0.01 \mathrm{~mol})$ or $\mathbf{1 2 b}(3.57 \mathrm{~g}, 0.01 \mathrm{~mol})$, in methanol $(50 \mathrm{~mL})$ was added the freshly distilled 2 nd amine, namely piperazine $(0.95 \mathrm{~mL}, 0.01 \mathrm{~mol})$ or morpholine $(0.86 \mathrm{~mL}$, $0.01 \mathrm{~mol})$. The reaction mixture was stirred under reflux for $7-9 \mathrm{~h}$, then allowed to cool to $0{ }^{\circ} \mathrm{C}$ for $14 \mathrm{~h}$ and the solid obtained was filtered, washed with water $(100 \mathrm{~mL})$ dried and recrystallized from appropriate solvent to produce $(\mathbf{1 4 a}-\mathbf{d})$.

\subsection{Synthesis of 7-Methoxy-6-methyl-1-(piperazin-1-yl)-3H-furo[3,2-g]pyrimido[1,6-a]quinazolin- 3-one (14a)}

The compound was obtained from the reaction of (12a) with piperazine, as pale yellow crystals, crystallized from dioxane in 79\% yield, m.p. $202{ }^{\circ} \mathrm{C}$. IR $\left(v, \mathrm{~cm}^{-1}\right) \mathrm{KBr}: 3390$ (br, NH), 3044 (CH-aryl), 2940 (CH-aliph), 1687 (CO, amide), 1620 (C=N). ${ }^{1} \mathrm{H}-\mathrm{NMR}$ (DMSO-d 6 , ppm) $\delta 2.29\left(\mathrm{~s}, 3 \mathrm{H}, \mathrm{CH}_{3}\right), 2.82-2.91$ $\left(\mathrm{m}, 8 \mathrm{H}\right.$, piperazine), $3.92\left(\mathrm{~s}, 3 \mathrm{H}, \mathrm{OCH}_{3}\right), 6.78(\mathrm{~d}, 1 \mathrm{H}, J=2.36 \mathrm{~Hz}$, furan), $7.38(\mathrm{~s}, 1 \mathrm{H}$, pyrimidine), $7.66(\mathrm{~s}$, $1 \mathrm{H}$, phenyl), $7.81\left(\mathrm{~d}, 1 \mathrm{H}, J=2.37 \mathrm{~Hz}\right.$, furan), 10.30 (brs, NH, $\mathrm{D}_{2} \mathrm{O}$ exchangeable); ${ }^{13} \mathrm{C}-\mathrm{NMR}\left(\mathrm{DMSO}-d_{6}\right) \delta$ $23.2\left(1 \mathrm{C}, \mathrm{CH}_{3}\right), 48.3\left(2 \mathrm{C}, 2 \mathrm{CH}_{2}\right.$, piperazine), $52.5\left(2 \mathrm{C}, 2 \mathrm{CH}_{2}\right.$, piperazine), $61.7\left(1 \mathrm{C}, \mathrm{OCH}_{3}\right), 91.8(1 \mathrm{C}, \mathrm{CH}$, phenyl), 98.4 (1C, CH, pyrimidine), 100.1, 105.3, 108.2, 144.6, 146.1, 153.8, 154.9, 160.3, 164.4, 168.1(10C, Ar-C), $169.3(1 \mathrm{C}, \mathrm{C}=\mathrm{O})$; $\mathrm{MS}(70 \mathrm{eV}, \%) \mathrm{m} / \mathrm{z} 365\left(\mathrm{M}^{+}, 83 \%\right)$; Anal. Calc. (Found) for $\mathrm{C}_{19} \mathrm{H}_{19} \mathrm{~N}_{5} \mathrm{O}_{3}$ (365.39): C, 62.46 (62.55); H, 5.24 (5.18); N, 19.17 (19.24).

\subsection{Synthesis of 7-Methoxy-6-methyl-1-morpholino-3H-furo[3,2-g]pyrimido[1,6-a]quinazolin-3-one (14b)}

The compound was obtained from the reaction of (12a) with morpholine, as yellow crystals, crystallized from methanol in 75\% yield, m.p. $171^{\circ} \mathrm{C}$. IR $\left(v, \mathrm{~cm}^{-1}\right) \mathrm{KBr}: 3040$ (CH-aryl), 2936 (CH-aliph), 1685 (CO, amide), $1622(\mathrm{C}=\mathrm{N}) .{ }^{1} \mathrm{H}-\mathrm{NMR}$ (DMSO-d $\left.d_{6} \mathrm{ppm}\right) \delta 2.30\left(\mathrm{~s}, 3 \mathrm{H}, \mathrm{CH}_{3}\right), 3.14-3.23(\mathrm{~m}, 8 \mathrm{H}$, morpholine), $3.90\left(\mathrm{~s}, 3 \mathrm{H}, \mathrm{OCH}_{3}\right), 6.76(\mathrm{~d}, 1 \mathrm{H}, J=2.34 \mathrm{~Hz}$, furan), 7.33 (s, 1H, pyrimidine), 7.64 (s, $1 \mathrm{H}$, phenyl), 7.80 (d, 1H, $J=2.35 \mathrm{~Hz}$, furan); ${ }^{13} \mathrm{C}-\mathrm{NMR}\left(\mathrm{DMSO}-d_{6}\right) \delta 23.1\left(1 \mathrm{C}, \mathrm{CH}_{3}\right), 51.5\left(2 \mathrm{C}, 2 \mathrm{CH}_{2}\right.$, morpholine), $55.8\left(2 \mathrm{C}, 2 \mathrm{CH}_{2}\right.$, morpholine), $61.9\left(1 \mathrm{C}, \mathrm{OCH}_{3}\right), 91.5(1 \mathrm{C}, \mathrm{CH}$, phenyl), $99.5(1 \mathrm{C}, \mathrm{CH}$, pyrimidine), 100.7, 105.6, 108.4, 144.7, 146.3, 153.9, 155.2, 160.7, 164.8, 168.9 (10C, Ar-C), 169.8 (1C, $\mathrm{C}=\mathrm{O})$; $\mathrm{MS}(70 \mathrm{eV}, \%) \mathrm{m} / \mathrm{z} 366\left(\mathrm{M}^{+}, 78 \%\right)$; Anal. Calc. (Found) for $\mathrm{C}_{19} \mathrm{H}_{18} \mathrm{~N}_{4} \mathrm{O}_{4}$ (366.38): C, 62.29 (62.37); $\mathrm{H}, 4.95$ (4.88); N, 15.29 (15.22).

\subsection{Synthesis of 7,11-Dimethoxy-6-methyl-1-(piperazin-1-yl)-3H-furo[3,2-g]pyrimido[1,6-a]quinazolin- 3-one (14c)}

The compound was obtained from the reaction of $(\mathbf{1 2 b})$ with piperazine, as yellowish crystals, crystallized from ethanol in 74\% yield, m.p. $184{ }^{\circ} \mathrm{C}$. IR $\left(v, \mathrm{~cm}^{-1}\right) \mathrm{KBr}: 3395$ (br, NH), 3048 (CH-aryl), 2944 (CH-aliph), 1689 (CO, amide), 1624 (C=N). ${ }^{1} \mathrm{H}-\mathrm{NMR}$ (DMSO-d $\left.d_{6}, \mathrm{ppm}\right) \delta 2.31$ (s, 3H, $\mathrm{CH}_{3}$ ), $2.85-2.94$ (m, $8 \mathrm{H}$, piperazine), $3.97\left(\mathrm{~s}, 6 \mathrm{H}, 2 \mathrm{OCH}_{3}\right), 6.81(\mathrm{~d}, 1 \mathrm{H}, J=2.32 \mathrm{~Hz}$, furan), $7.36(\mathrm{~s}, 1 \mathrm{H}$, pyrimidine), 7.84 (d, $1 \mathrm{H}, J=2.30 \mathrm{~Hz}$, furan), 10.35 (brs, NH, $\mathrm{D}_{2} \mathrm{O}$ exchangeable); ${ }^{13} \mathrm{C}-\mathrm{NMR}$ (DMSO- $\left.d_{6}\right) \delta 23.5\left(1 \mathrm{C}, \mathrm{CH}_{3}\right)$, $48.6\left(2 \mathrm{C}, 2 \mathrm{CH}_{2}\right.$, piperazine), $52.8\left(2 \mathrm{C}, 2 \mathrm{CH}_{2}\right.$, piperazine), $61.8\left(2 \mathrm{C}, 2 \mathrm{OCH}_{3}\right), 99.6(1 \mathrm{C}, \mathrm{CH}$, pyrimidine $)$, 
100.5, 105.7, 108.9, 122.3, 130.2, 146.4, 146.5, 151.8, 154.1, 164.8, 168.5 (10C, Ar-C), 169.7(1C, C=O); MS

$(70 \mathrm{eV}, \%) \mathrm{m} / z 395\left(\mathrm{M}^{+}, 76 \%\right)$; Anal. Calc. (Found) for $\mathrm{C}_{20} \mathrm{H}_{21} \mathrm{~N}_{5} \mathrm{O}_{4}$ (395.42): $\mathrm{C}, 60.75$ (60.82); $\mathrm{H}, 5.35$

(5.43); N, 17.71 (17.78).

\subsection{Synthesis of 7,11-Dimethoxy-6-methyl-1-morpholino-3H-furo[3,2-g]pyrimido[1,6-a]quinazolin-} 3-one (14d)

The compound was obtained from the reaction of $(\mathbf{1 2 b})$ with morpholine, as yellowish crystals, crystallized from n-hexane in $72 \%$ yield, m.p. $156^{\circ} \mathrm{C}$. IR $\left(v, \mathrm{~cm}^{-1}\right) \mathrm{KBr}: 3039$ (CH-aryl), 2934 (CH-aliph), 1683 (CO, amide), $1626(\mathrm{C}=\mathrm{N}) .{ }^{1} \mathrm{H}-\mathrm{NMR}$ (DMSO-d $\left.d_{6} \mathrm{ppm}\right) \delta 2.32\left(\mathrm{~s}, 3 \mathrm{H}, \mathrm{CH}_{3}\right), 3.16-3.25(\mathrm{~m}, 8 \mathrm{H}$, morpholine), $3.97\left(\mathrm{~s}, 6 \mathrm{H}, 2 \mathrm{OCH}_{3}\right), 6.79(\mathrm{~d}, 1 \mathrm{H}, \mathrm{J}=2.33 \mathrm{~Hz}$, furan), 7.37 (s, 1H, pyrimidine), 7.82 (d, $1 \mathrm{H}, J=2.37 \mathrm{~Hz}$, furan); ${ }^{13} \mathrm{C}-\mathrm{NMR}\left(\mathrm{DMSO}-d_{6}\right) \delta 23.7\left(1 \mathrm{C}, \mathrm{CH}_{3}\right), 53.8\left(2 \mathrm{C}, 2 \mathrm{CH}_{2}\right.$, morpholine), $57.6(2 \mathrm{C}$, $2 \mathrm{CH}_{2}$, morpholine), $61.9\left(2 \mathrm{C}, 2 \mathrm{OCH}_{3}\right), 99.8$ (1C, $\mathrm{CH}$, pyrimidine), 100.4, 105.3, 108.7, 122.6, 130.1, 146.2, 146.8, 151.3, 154.2, 164.5, 168.6 (11C, Ar-C), 169.4 (1C, C=O); MS (70 eV, \%) m/z 396 (M+ , $74 \%)$; Anal. Calc. (Found) for $\mathrm{C}_{20} \mathrm{H}_{20} \mathrm{~N}_{4} \mathrm{O}_{5}$ (396.40): C, 60.60 (60.68); H, 5.09 (5.16); N, 14.13 (14.20).

\subsection{Biological Screening}

The antimicrobial activity of the newly prepared compounds was tested in vitro against Gram-positive bacteria Staphylococcus aureus (ATCC ${ }^{\circledR} 6538^{\mathrm{TM}}$ ), Streptococcus pyogenes (ATCC ${ }^{\circledR} 19615^{\mathrm{TM}}$ ), Gram-negative bacteria Escherichia coli $\left(\mathrm{ATCC}^{\circledR} 25922^{\mathrm{TM}}\right.$ ), Klebsiella pneumoniae (ATCC ${ }^{\circledR} 10031^{\mathrm{TM}}$ ) and the fungi Aspergillus niger (ATCC ${ }^{\circledR} 16888^{\mathrm{TM}}$ ), Alternaria alternate, Curvularia lunata and Candida albicans $\left(\mathrm{ATCC}^{\circledR} 10231^{\mathrm{TM}}\right.$ ). The newly prepared compounds were dissolved in dimethyl sulfoxide (DMSO) and tested for their antimicrobial activity by the agar disk diffusion technique. Cefotaxime sodium and nystatin [34,35] were used as the standard drugs for antibacterial and antifungal assays, respectively. A solution of $100 \mu \mathrm{g} \mathrm{mL}^{-1}$ of the tested compound was practical and microplate-wells, $1 \mathrm{~cm}$ in diameter, were used. Zones of inhibition were measured with calipers or automated scanners and were paralleled with those of the standards. Cefotaxime sodium $\left(0.15 \mu \mathrm{mol} \mathrm{mL}^{-1}\right)$ and nystatin $\left(0.037 \mu \mathrm{mol} \mathrm{mL}{ }^{-1}\right)$ were used as the standard drugs for antibacterial and antifungal activity, respectively. Compound-impregnated disks were placed on an agar plate containing a standard suspension of microorganisms. The plate was incubated for $24 \mathrm{~h}$ at $37^{\circ} \mathrm{C}$. For the assessment of the minimum inhibitory concentration (MIC) by the serial plate dilution way [34,35], $5 \mathrm{mg}$ of each tested compound was dissolved in $1 \mathrm{~mL}$ of DMSO separately to prepare stock solutions. Serial dilutions were prepared from each stock solution. The plates were incubated at $37^{\circ} \mathrm{C}$ for $24 \mathrm{~h}$. MIC is defined as the lowest concentration ( $\mu \mathrm{mol} \mathrm{mL}{ }^{-1}$ ) of the tested compound that results in no visible growth on the plates. DMSO was used as the solvent control to ensure that the solvent had no effect on bacterial growth. The results are shown in Tables 1 and 2.

\section{Conclusions}

In this work, we synthesized novel heterocyclic compounds with potent antimicrobial activity starting from furochromones (visnagenone $\mathbf{2 a}$ or khellinone $\mathbf{2 b}$ ). New derivatives were prepared in good yields such as; furopyrimido quinazolinones, furothiazolo pyrimido quinazolinones, substitutedbenzyliden-furothiazolo pyrimido quinazolinones, pyrazolo furopyrimido quinazolin-ones, oxo or thioxo-pyrimidin-furopyrimido quinazolinones and methylthio, methylsulfonyl, piperazino or morpholino-furopyrimido quinazolinones. Some new prepared compounds such as substitutedbenzylidene-furothiazolo pyrimido quinazolinones $8 \mathbf{a}-\mathbf{f}$ showed higher activity against the tested microorganisms (bacteria and fungi).

Funding: This research received no external funding.

Acknowledgments: The author is extremely grateful to Department of Chemistry of Natural and Microbial Products, National Research Centre for helping us to screen the newly synthesized compounds for antimicrobial activity. 
Conflicts of Interest: The author a declare no conflict of interest.

\section{References}

1. Trease, G.E.; Evans, W.C. Pharmacognosy, 12th ed.; Bailliere Tindall: Eastbourne, UK, 1983; p. 385.

2. Wallis, T.E. Textbook of Pharmacognosy, 5th ed.; Whitefriars: London, UK, 1967; p. 244.

3. El-Domiaty, M.M. Improved high-performance liquid chromatographic determination of khellin and visnagin in Ammi visnaga fruits and pharmaceutical formulations. J. Pharm. Sci. 1992, 81, 475-478. [CrossRef] [PubMed]

4. Bhagavathula, A.S.; Al-Khatib, A.J.M.; Elnour, A.A.; Kalbani, N.M.S.A.; Shehab, A. Ammi visnaga in treatment of urolithiasis and hypertriglyceridemia. Pharmacogn. Res. 2015, 7, 397-400. [CrossRef]

5. Gammill, R.B.; Day, C.E.; Schurr, P.E. Khellin analogues. 1. General topological requirements for lipid-altering activity in furochromones. J. Med. Chem. 1983, 26, 1672-1674. [CrossRef] [PubMed]

6. De Leeuw, J.; Assen, Y.; Van Der Beek, N.; Bjerring, P.; Martino Neumann, H. Treatment of vitiligo with khellin liposomes, ultraviolet light and blister roof transplantation. J. Eur. Acad. Dermatol. Venereol. 2011, 25, 74-81. [CrossRef] [PubMed]

7. Abdou, S.E.; El-Qusy, S.M.; Ghabrial, S.S.; Haggag, M.I. Reactions with visnaginone: Synthesis, cyclisation and microbial evaluation of some visnaginone thiosemicarbazone derivatives. Mod. App. Sci. 2011, 5. [CrossRef]

8. El-Nakkady, S.S.; Roaiah, H.F.; El-Serwy, W.S.; Soliman, A.M.; El-Moez, S.I.; Abdel-Rahman, A.A. Antitumor and antimicrobial activities of some hetero aromatic benzofurans derived from naturally occurring visnagin. Acta Pol. Pharm. 2012, 69, 645-655. [PubMed]

9. Kandil, A.; Gobran, W.; Samaan, H.A.; Abu-Shady, H. The spasmolytic potential of a new khellin derivative. J. Drug Res. 1977, 9, 35.

10. Oguro, K.; Kubota, K.; Kimura, T.; Hashimoto, K. Effects of various coronary vasodilators on myocardial oxygen consumption. Jpn. J. Pharmacol. 1973, 23, 459-466. [CrossRef] [PubMed]

11. Oguro, K.; Hashimoto, K. Quanttative and comparative studies of pharma-cological features in the coronary, femoral and renal circulations with different coronary vasodilators. Jpn. J. Pharmacol. 1974, 24, 227-233. [CrossRef] [PubMed]

12. Vedaldi, D.; Caflleri, S.; Dall'Acqua, F.; Andrea, L.; Bovalini, L.; Martelli, P. Khellin, a naturally occurring furochromone, used for the photochemotherapy of skin diseases: Mechanism of action. Farmaco 1988, 4, 333-346.

13. Trabalzini, L.; Martelli, P.; Bovalini, L.; Dall'Acqua, F.; Sage, E. Photosensitization of DNA of defined sequence by furochromones, Khellin and Visnagen. J. Photochem. Photobiol. B 1990, 7, 317-336. [CrossRef]

14. Elgazwy Abdel-Sattar, S.H.; Edrees Mastoura, M.; Ismail Nasser, S.M. Molecular modeling study bioactive natural product of khellin analogues as a novel potential pharmacophore of EGFR inhibitors. J. Enzym. Inhib. Med. Chem. 2013, 28, 1171-1181. [CrossRef] [PubMed]

15. Melzig, L.; Rauhut, C.B.; Knochel, P. 2, 3-Functionalization of furans, benzofurans and thiophenes via magnesiation and sulfoxide-magnesium exchange. Chem. Commun. 2009, 3536-3538. [CrossRef] [PubMed]

16. Mustafa, A. Furochromones. In Furopyrans and Furopyrones; John Wiley and Sons: London, UK; New York, NY, USA; Sydney, Australia, 1967; pp. 102-159.

17. Gammill, R.B.; Hyde, B.R. Total synthesis of the lipid-altering and antiatherosclerotic furochromone khellin. The furoic acid route to highly functionalized benzofurans. J. Org. Chem. 1983, 48, 3863-3865. [CrossRef]

18. Galal, S.A.; Abd EL-All, A.S.; Abdallah, M.M.; EL-Diwani, H.I. Synthesis of potent antitumor and antiviral benzofuran derivatives. Bioorg. Med. Chem. Lett. 2009, 19, 2420-2428. [CrossRef] [PubMed]

19. Kim, S.; Salim, A.A.; Swanson, S.M.; Kinghorn, A.D. Potential of cyclopenta[b]benzofurans from Aglaia species in cancer chemotherapy. Anticancer Agents Med. Chem. 2006, 6, 319-345. [CrossRef] [PubMed]

20. Hudson, J.B.; Towers, G.H.N. Phytomedicines as antivirals. Drugs Future 1999, 24, 295-320. [CrossRef]

21. Abu-Hashem, A.A.; Youssef, M.M. Synthesis of new visnagen and khellin furochromone pyrimidine derivatives and their anti-inflammatory and analgesic activity. Molecules 2011, 16, 1956-1972. [CrossRef] [PubMed]

22. Abu-Hashem, A.A.; El-Shazly, M. Synthesis of new quinoxaline, pyrimidine, and pyrazole furochromone derivatives as cytotoxic agents. Monatsh. Chem. 2017, 148, 1853-1863. [CrossRef] 
23. Keshk, E.M.; Abu-Hashem, A.A.; Girges, M.M.; Abdel-Rahman, A.H.; Badria, F.A. Synthesis of benzo[1,2-b:5,4-b]difuranyl-triazoles, oxadiazoles, thiazolidione, thiadiazoles, and the use of DNA in evaluation of their biological activity. Phosphorus Sulufur Silicon Relat. Elem. 2004, 179, 1577-1593. [CrossRef]

24. Kamal, A.; Shankaraiah, N.; Prabhakar, S.; Reddy, C.R.; Markandeya, N.; Reddy, K.L.; Devaiah, V. Solid-phase synthesis of new pyrrolobenzo diazepine-chalcone conjugates: DNA-binding affinity and anticancer activity. Bioorg. Med. Chem. Lett. 2008, 18, 2434-2439. [CrossRef] [PubMed]

25. Abd Elhafez, O.M.; El Khrisy Eel, D.; Badria, F.; Fathy Ael, D. Synthesis and biological investigation of new thiazolidinone and oxadiazoline coumarin derivatives. Arch. Pharm. Res. 2003, 26, 686-696. [CrossRef] [PubMed]

26. Wenzel, N.I.; Chavain, N.; Wang, Y.; Friebolin, W.; Maes, L.; Pradines, B.; Lanzer, M.; Yardley, V.; Brun, R.; Herold-Mende, C.; et al. Antimalarial versus cytotoxic properties of dual drugs derived from 4-aminoquinolines and Mannich bases: Interaction with DNA. J. Med. Chem. 2010, 53, 3214-3226. [CrossRef] [PubMed]

27. Scozzafava, A.; Owa, T.; Mastrolorenzo, A.; Supuran, C.T. Anticancer and antiviral sulfonamides. Curr. Med. Chem. 2003, 10, 925-953. [CrossRef] [PubMed]

28. Rescifina, A.; Chiacchio, M.A.; Corsaro, A.; De Clercq, E.; Iannazzo, D.; Mastino, A.; Piperno, A.; Romeo, G.; Romeo, R.; Valveri, V. Synthesis and biological activity of isoxazolidinyl polycyclic aromatic hydrocarbons: Potential DNA intercalators. J. Med. Chem. 2006, 49, 709-715. [CrossRef] [PubMed]

29. Abu-Hashem, A.A.; El-Shazly, M. Synthesis, reactions and biological activities of furochromones: A review. Eur. J. Med. Chem. 2015, 90, 633-665. [CrossRef] [PubMed]

30. El-Gazzar, A.B.A.; Youssef, A.M.S.; Youssef, M.M.; Abu-Hashem, A.A.; Badria, F.A. Design and synthesis of azolopyrimidoquinolines, pyrimidoquinazolines as anti-oxidant, anti-inflammatory and analgesic activities. Eur. J. Med. Chem. 2009, 44, 609-624. [CrossRef] [PubMed]

31. Riyadh, S.M.; Farghaly, T.A.; Gomha, S.M. Novel polyazaheterocyclic systems: Synthesis, antitumor, and antimicrobial activities. Arch. Pharm. Res. 2010, 33, 1721-1728. [CrossRef] [PubMed]

32. Ali, T.E.; Ibrahim, M.A. Synthesis and Antimicrobial Activity of Chromone-linked 2-Pyridone Fused with 1,2,4-Triazoles, 1,2,4-Triazines and 1,2,4-Triazepines Ring Systems. J. Braz. Chem. Soc. 2010, 21, 1007-1016. [CrossRef]

33. Khalil, A.M.; Berghot, M.A.; Gouda, M.A. Synthesis and antibacterial activity of some new heterocycles incorporating phthalazine. Eur. J. Med. Chem. 2009, 44, 4448-4454. [CrossRef] [PubMed]

34. Jain, S.R.; Kar, A. The antibacterial activity of some essential oils and their combinations. Planta Med. 1971, 20, 118-123. [CrossRef] [PubMed]

35. Abu-Hashem, A.A. Synthesis and biological activity of pyrimidines, quinolines, thiazines and pyrazoles bearing a common thieno moiety. Acta Pol. Pharm. Drug Res. 2018, 75, 59-70.

Sample Availability: Samples of the synthesized compounds are available from the authors.

(C) 2018 by the author. Licensee MDPI, Basel, Switzerland. This article is an open access article distributed under the terms and conditions of the Creative Commons Attribution (CC BY) license (http:/ / creativecommons.org/licenses/by/4.0/). 